\title{
Propeller Cavitation Noise Investigations of a Research Vessel Using Medium Size Cavitation Tunnel Tests and Full-Scale Trials
}

Batuhan Aktas ${ }^{1, *}$, Mehmet Atlar ${ }^{1}$, Serkan Turkmen ${ }^{1}$, Weichao Shi ${ }^{1}$, Roderick Sampson ${ }^{1}$, Emin Korkut ${ }^{2}$, Patrick Fitzsimmons $^{1}$

${ }^{1}$ School of Marine Science and Technology, Newcastle University, Newcastle upon Tyne NE1 7RU, UK

${ }^{2}$ Istanbul Technical University, Faculty of Naval Architecture and Ocean Engineering, 34469 Maslak-Istanbul, Turkey

\section{Corresponding Author:}

*Batuhan Aktas, b.aktas@newcastle.ac.uk

School of Marine Science and Technology

Armstrong Building, Newcastle University

United Kingdom, NE1 7RU

Tel: $0044(0) 1912226726$

Fax: $0044(0) 1912225491$

\section{Abstract}

The rising environmental awareness of various adverse emissions by commercial shipping has recently targeted Underwater Radiated Noise (URN) due to its potential impact on marine mammals. Amongst the various sources on-board a commercial ship, cavitation is the dominating one following its inception. In order to ensure acceptable noise levels for sustainable shipping, accurate prediction of the noise signature is vital. Within this framework, a widely utilized method for full-scale noise prediction is to conduct model tests in cavitation tunnels and to extrapolate to full-scale.

The aim of this paper is to provide invaluable URN data of a full-scale vessel and its prediction using cavitation tests from a medium-sized tunnel to evaluate the prediction methodology. Extrapolated URN data based on the tunnel tests was compared with the data obtained from the full-scale trials with The Princess Royal in order to assess the prediction methodology. The comparisons indicate that, whilst the ideal experimental approach is to conduct such involving tests with a full-hull model in large cavitation tunnels, the medium size facilities using dummy-hull models with wake screens, can still provide a very useful means for the URN investigations with a rapid turn around and an economical way of conducting such tests.

Keywords: Underwater Radiated Noise; Propeller Cavitation Noise; Experimental Hydrodynamics; Cavitation Tunnel Noise Predictions 
Over the past half century the volume of commercial shipping has experienced an increasing trend due to increasing ship size, service speed and number of ships operating (Hildebrand, 2009). This trend has resulted in significant elevation of various emissions by the seagoing vessels. In order to ensure sustainable shipping, various anthropogenic impacts have been targeted by environmentalists, in particular, Underwater Radiated Noise (URN) from commercial shipping. URN has been known to propagate over long distances from the source, especially at low frequencies where the propagation losses are small due to the low sound absorption rate of the water medium (Fisher \& Simmons, 1977). The low frequency band (10 to $100 \mathrm{~Hz}$ ) for ships contains the tonal frequency components such as propeller Blade Passing Frequencies (BPF) and engine firing frequencies (Zoet et al., 2013). This low frequency band may overlap and mask the communication frequency band of marine mammals and hence adversely affecting their fundamental living activities (Hildebrand, 2005). Originating from these concerns the international organizations and committees, such as International Maritime Organization (IMO) and Marine Environmental Protection Committee (MEPC) (IMO, 2011; MEPC, 2009) have made calls and initiated activities to study the URN from commercial shipping to help in the development of potential guidelines and regulations. Moreover, the EU has established the Marine Strategy Framework Directive (MSFD) to investigate and implement programmes of measures which are designed to achieve or maintain 'Good Environmental Status' in the marine environment (Van der Graaf et al., 2012).

Shipping noise originates from various sources on board a vessel. Amongst these sources, propeller cavitation usually dominates the overall radiated noise spectrum above the inception threshold (Ross, 1987). In other words after cavitation inception and well developed cavitation is experienced by the propeller, a significant increase in level occurs across the entire frequency band of the URN spectrum (Abrahamsen, 2012; Arveson \& Vendittis, 2000). In turn, the cavitation noise overshadows the other contributing sources and dominates the overall URN spectra. Whilst it is not possible to avoid cavitation for efficient commercial ships at service speed conditions, various full-scale URN measurements have shown room for improvement. Among the same type of vessels full scale measurements have shown up to $20 \mathrm{~dB}$ difference in the measured noise levels (McKenna et. Al., 2012; MCR, 2011; Wales \& Heitmeyer, 2002). This may suggest that the current practice of ship design can be further scrutinized in terms of the URN characteristics of the ships and hence may lead to minimizing the impact on ever-increasing ambient noise levels in the world's oceans (Renilson Marine Consulting Pty Ltd, 2009). 
In order to tackle the above concerns a reliable and accurate prediction of URN at an early design stage of ships is essential.

The current state of the art methods for the URN predictions utilize experimental methods based mainly on cavitation tunnels, semi-empirical methods based on statistical databases and Computational Fluid Dynamics (CFD) methods with significant simplifications. Although computational power has been increasing at an exponential pace, URN prediction using the CFD method is at its infancy and requires coupling of as many as five different codes as reported e.g. in (Wijngaarden, 2005). While recent developments in the CFD field, which are based on the incompressible Reynolds Averaged Navier Stokes (RANS) equations coupled with the Ffowcs-Williams Hawkings (F-HW) equation, have made the URN predictions possible, this approach still needs further development in terms of the accuracy of the predictions as well as the cost of the computational resources (ITTC, 2014). Semi empirical methods are based on a number of different approaches; some being developed based on data from the World War II period. These early methods over predict the URN levels of preset-day commercial ships (Okamura \& Asano, 1988) due to developments in the field of propeller design (Brown, 1976; Ross, 1987). More recently introduced semi-empirical methods are commercially confidential, being based on simple models for which various coefficients have been derived from large databases of full scale noise measurements (e.g. Raestad, 1996). By considering the additional complexities associated with the numerical modelling of the propeller cavitation, perhaps, the most reliable current prediction method may be that based on the experimental methods (ITTC, 2014)

Outside the research vessel and naval communities, publicly available information is scare for both full-scale URN levels and cavitation tunnel based predictions. Furthermore such data, which are publicly available, are either unsatisfactory due to a lack of information on the main particulars of target vessels and their operating conditions and/or measurement technique and methods used to collect the data (Bark, 1985). Allied with this unfavorable status the complementary information, which can enhance the understanding of the noise emitting mechanisms such as cavitation observations, pressure pulse measurements, is even more scarce thus creating a large gap in the current state of the art.

Within the above framework, in complementing the European R\&D activities on the subject of URN from commercial shipping, several collaborative European R\&D projects have been underway (e.g. SILENV, AQUO, SONIC) under the $7^{\text {th }}$ Framework Programme (FP) of the EU. Amongst them SONIC (Suppression Of Noise Induced by Cavitation) has brought together 12 world-leading hydrodynamic institutes, noise experts, propeller designers, universities, European shipyards and marine biologists to develop guidelines to assist in regulating the underwater noise emitted by shipping in the North Sea 
1 (SONIC, 2012). The project participants have developed techniques to model cavitation noise computationally and

2 experimentally, for use by vessel operators to monitor the shipping noise. The participants have also mapped the spatial

3 distribution of the URN caused by a single ship (a noise footprint) and sets of ships in an area (a noise map). To do this, the project partners have been measuring ship radiated noise both on and off board to test these measurements in full-scale trials at sea. Ultimately, the SONIC project will contribute to validating different prediction methods for ship URN and to developing cavitation noise models to help the classification and regulations of ships based on their noise footprints. In the long term, such information is also expected to assist in the development of guidelines for the future design of low-noise ships.

As part of the SONIC project activities, the prediction of full-scale URN spectra has been investigated based on model tests in different sizes and types of experimental facilities. In those investigations, Newcastle University was involved in a fullscale trial campaign conducted jointly by the project partners to measure the URN levels from the research catamaran "The Princess Royal". The full-scale trial campaign was conducted in the North East coast region of England (Blyth) in September 2013 and involved various on-board and off-board measurements. The campaign included the collection of the URN data from suspended arrays of hydrophones, fluctuating hull pressures, vibration pick-ups and propeller cavitation observations. Following the trials, some of these full-scale runs were simulated experimentally in the medium size cavitation, "The Emerson Cavitation Tunnel", by using a 1:3.5 scale dummy model of the starboard demi-hull. The experimentally measured URN levels were extrapolated using the ITTC procedure (ITTC, 1987) to compare with the fullscale measurements. The comparisons also included the cavitation observations in both the full-scale and model scale.

Based upon the above background the aim of this paper is to provide invaluable URN data from a full-scale target vessel and its prediction using medium size cavitation tunnel tests to evaluate the prediction methodology by making use of the data generated as part of the collaborative SONIC project activities.

In order to satisfy the above aim, following this introduction, Section 2 of the paper describes the experimental facilities and set-up including the Emerson Cavitation Tunnel (2.1), general particulars of The Princess Royal and her propellers (2.2), and details of the wake simulation activities (2.3) as part of the model test procedures. In Section 3, the selected fullscale trial runs and corresponding tunnel test conditions are described. Section 4 presents the details of the noise measurements. This include a description of the equipment and analysis procedures as well extrapolation of the noise characteristics to the full-scale for comparisons with the full-scale noise data (4.1). The results are presented in section (4.2) 


\section{Experimental facilities and setup}

\subsection{The Emerson Cavitation Tunnel}

3 The experiments were carried out in the Emerson Cavitation Tunnel (ECT) of Newcastle University, which has a measuring section of $3.1 \mathrm{~m} \times 1.21 \mathrm{~m} \times 0.8 \mathrm{~m}(\mathrm{LxBxH})$, as shown in Figure 1 and Figure 2. More detailed information about the tunnel and its details can be found in (Atlar, 2011).

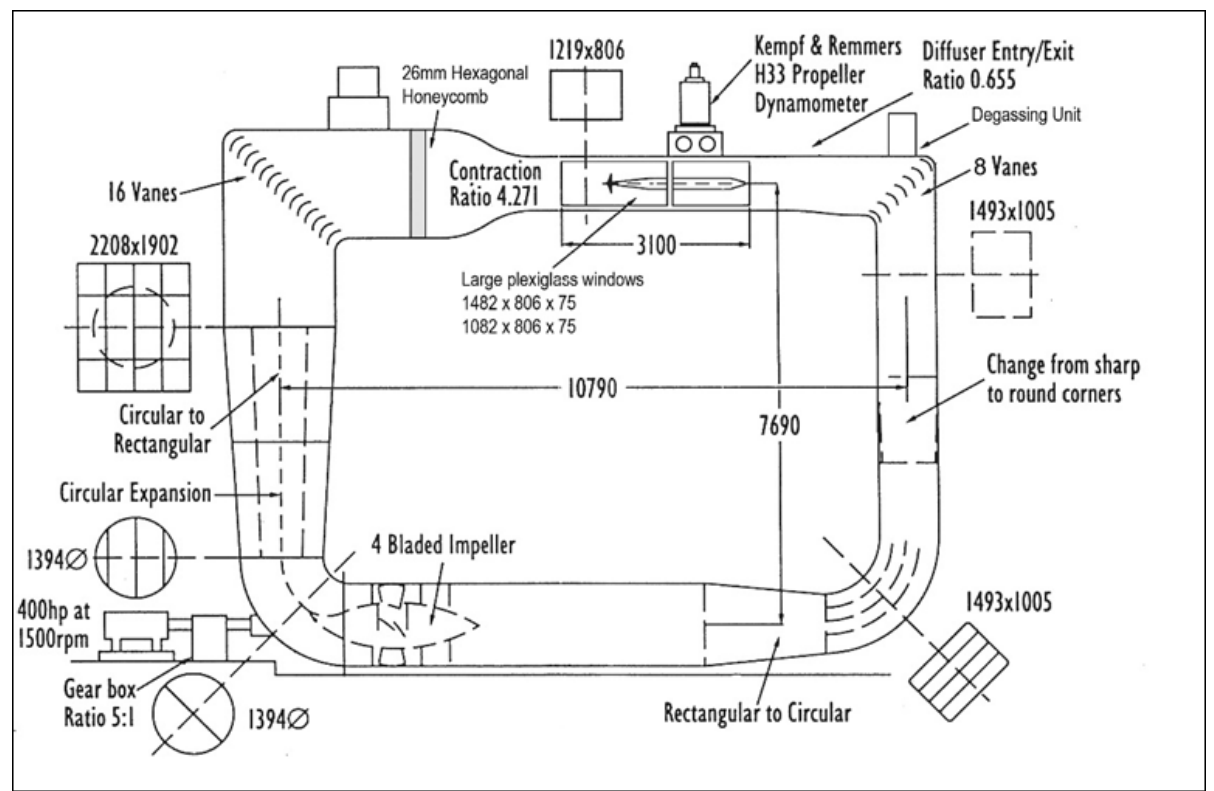

Figure 1: Sketch of Emerson Cavitation Tunnel

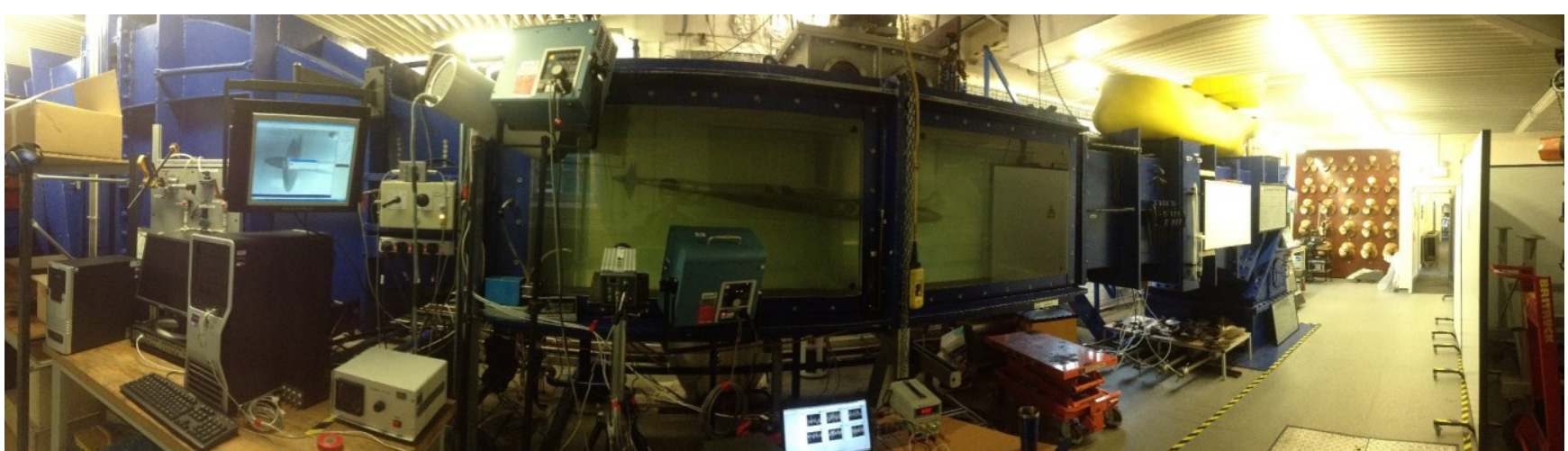

Figure 2: A panoramic view of Emerson Cavitation Tunnel and test set-up

\subsection{Main particulars of The Princess Royal and propeller}

The Princess Royal is a displacement type of Deep-V catamaran, which was designed in-house and built locally, as described in detail by (Atlar et al 2013). During the experiments, the starboard demi-hull of the vessel was used as a basis for simulating the hull wake based on the well-known "dummy-hull" approach usually adopted in small and medium size 
cavitation tunnels. The model scale factor of 1:3.5 was set by considering various limiting factors such as avoiding an undesirable blockage effect, achieving a reasonable Reynolds number range for minimizing the scale effects and achieving a respectable size for avoiding practical size limitation. At this scale, the demi-hull was too long $(5.39 \mathrm{~m})$ to fit the tunnel's test section and had to be truncated down to $3 \mathrm{~m}$. The truncation was carried out in the parallel mid-section between the properly represented fore and aft sections. This is a well-recognized approach, which is used throughout the industry. The chosen dimensions combined with the set tunnel conditions resulted in a propeller Reynolds number ranging from $8.67 \times 10^{5}$ to $1.47 \times 10^{6}$. The largest blockage of the tunnel cross section was $16.5 \%$ which is well within the ITTC guidelines (ITTC, 2011). Based on the above justification; the comparative general specifications of the dummy-hull model and the full-scale vessel are given in Table 1.

Table 1 : Full-scale vessel and dummy-hull model particulars

\begin{tabular}{|l|c|c|c|}
\hline \multicolumn{1}{|c|}{ Main particulars } & $\begin{array}{c}\text { Full scale } \\
\text { (Start of Trials) }\end{array}$ & $\begin{array}{c}\text { Model scale } \\
\text { Full scale } \\
\text { (End of Trials) }\end{array}$ & $\begin{array}{c}\text { (Dummy model of } \\
\text { Starboard Hull) }\end{array}$ \\
\hline Length overall & 18.88 & - & 3.007 \\
\hline Length between perpendiculars, $\mathrm{L}_{\mathrm{PP}}(\mathrm{m})$ & 16.45 & - & N/A \\
\hline Length of waterline, L $\mathrm{WL}_{\mathrm{W}}(\mathrm{m})$ & 16.45 & - & 0.558 \\
\hline Beam, moulded, B (m) & 7.3 & - & 0.448 \\
\hline Draft at forward perpendicular, $\mathrm{T}_{\mathrm{F}}(\mathrm{m})$ & 1.745 & 1.72 & 0.557 \\
\hline Draft at aft perpendicular, $\mathrm{T}_{\mathrm{A}}(\mathrm{m})$ & 1.845 & 1.82 & 0.262 \\
\hline Propeller distance from aft perpendicular $(\mathrm{m})$ & 0.9 & - & 1 \\
\hline Number of propellers, NP & 2 & 2 & . \\
\hline
\end{tabular}

The data in Table 1 correspond to two loading conditions of the vessel, namely, at the start and end of the full-scale trials. This was because the trials were conducted over three consecutive days meaning the fuel consumptions and various other changes on-board resulted in slight changes in the loading and hence running conditions of the vessel. The loading conditions of the vessel were taken from the logbook of the vessel and then used as an input to the stability booklet to interpolate the draft readings for the corresponding loading. The procedure was then repeated taking into account the fuel consumption during the course of the trials. The draft values in Table 1 represent only the static trim condition. Further manipulation of this data to take account of the dynamic trim is discussed in Section 3. Based on the selected scale ratio the main characteristics of the model and full-scale propeller are given in Table 2. 
Table 2: Full-scale propeller and model propeller particulars

\begin{tabular}{|c|c|c|}
\hline & In full scale & In model scale \\
\hline Propeller diameter, D (m) & 0.75 & 0.214 \\
\hline Number of blades, $Z$ & \multicolumn{2}{|c|}{5} \\
\hline Direction of rotation & $\begin{array}{l}\text { Port: left turning - outwards } \\
\text { SB: right turning - outwards }\end{array}$ & SB: right turning - outwards \\
\hline Type of propeller & \multicolumn{2}{|c|}{ Fixed pitch } \\
\hline Pitch ratio at $0.7 \mathrm{R} P / \mathrm{D}$ & \multicolumn{2}{|c|}{1.057} \\
\hline Chord length at $0.7 \mathrm{R},(\mathrm{m})$ & 0.352 & 0.1006 \\
\hline Skew angle, $\theta_{\mathrm{S}}(\mathrm{Deg})$ & \multicolumn{2}{|c|}{$19^{\circ}$} \\
\hline Rake angle (Deg)_ & \multicolumn{2}{|c|}{$0^{\circ}$} \\
\hline Expanded Blade Area Ratio, EAR & \multicolumn{2}{|c|}{1.057} \\
\hline Boss diameter ratio, $\mathrm{D}_{\mathrm{Hub}} / \mathrm{D}$ & \multicolumn{2}{|c|}{0.2} \\
\hline Scale ratio, $\lambda$ & \multicolumn{2}{|c|}{3.5} \\
\hline
\end{tabular}

2

\subsection{Wake simulations}

In cavitation tunnel tests, the preference is to use a full-hull model of the subject vessel. However due to tunnel size limitations the use of so-called "dummy-hull model with additional wake mesh" is another practical approach which has been used in many medium size cavitation tunnels including the Emerson Cavitation Tunnel. This approach has the advantage of better representing the 3D flow effects compared to a simple 2D wake screen approach, although the latter is more economical in time and cost. Based on this rationale the starboard demi-hull of The Princess Royal was modelled by properly scaling the fore and after body sections of the demi-hull while truncating the middle part in between these two sections. Furthermore, 2D wake screens were added at the aft end to account for the flow retardation lost by truncation of the hull in the middle section as shown in Figure 3. The density of the screen meshes was adjusted through an iterative wake simulation exercise by using the stereoscopic Particle Image Velocimetry (PIV) system of the ECT. The target wake for these simulations was based on the wake measurements conducted with a 1:5 full-model of The Princess Royal in the Istanbul Technical University towing tank excluding the rudder and interceptors (Korkut \& Takinaci, 2013) 


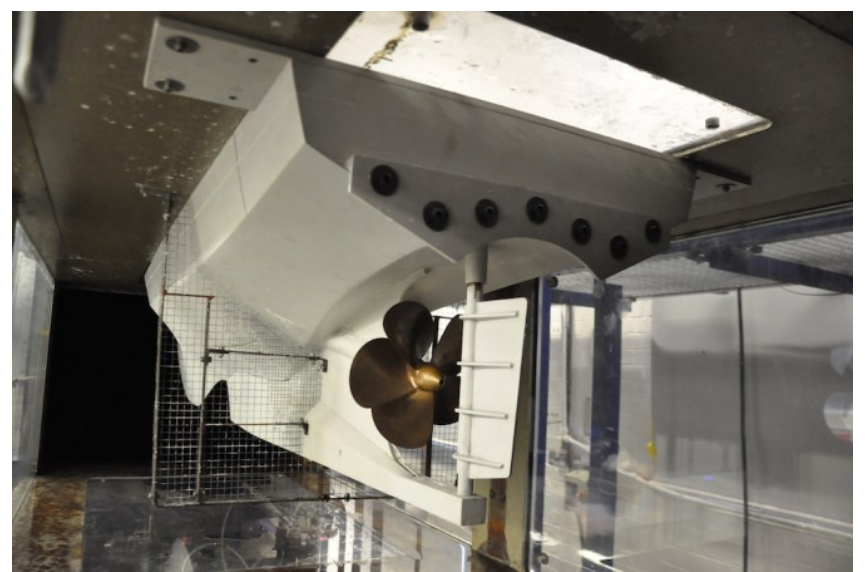

Figure 3 : Dummy-hull model fitted in Emerson Cavitation Tunnel

Figure 4 shows the contour plots of the target wake and simulated wake while Figure 5 and Figure $\mathbf{6}$ show the comparative

radial plots of the wake velocities at two critical outer radius. As shown in the two latter figures reasonable resemblance has

simulations, for more representative cavitation tests.
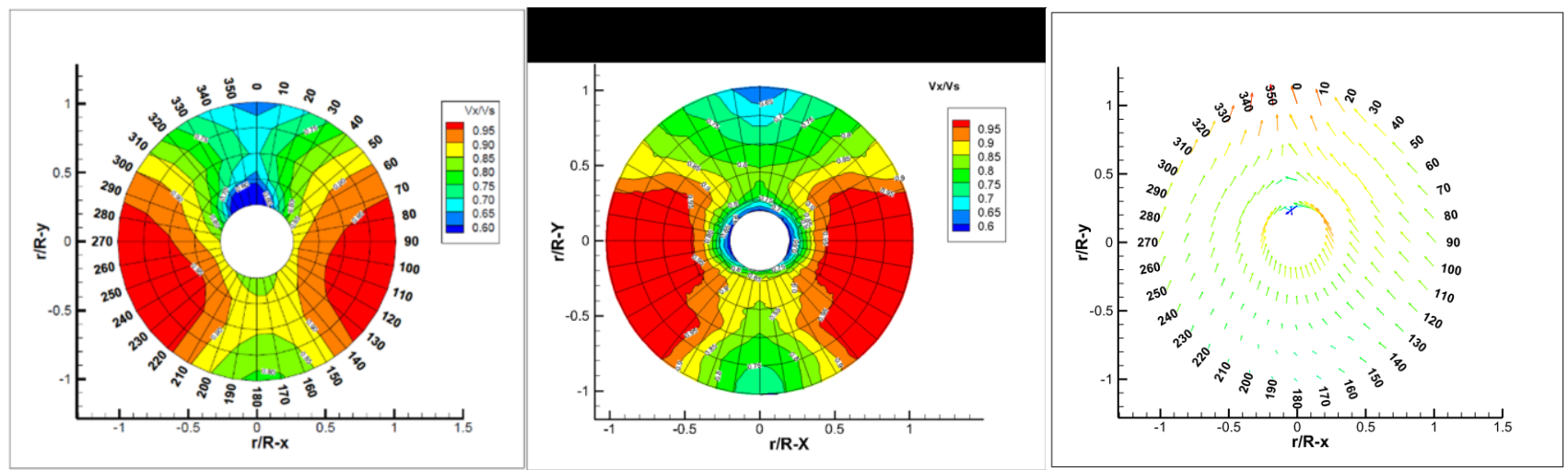

Figure 4 : Contour plots of target wake (left) and simulated wake (middle) together with the vector plot of the radial and tangential components of the target wake (right)

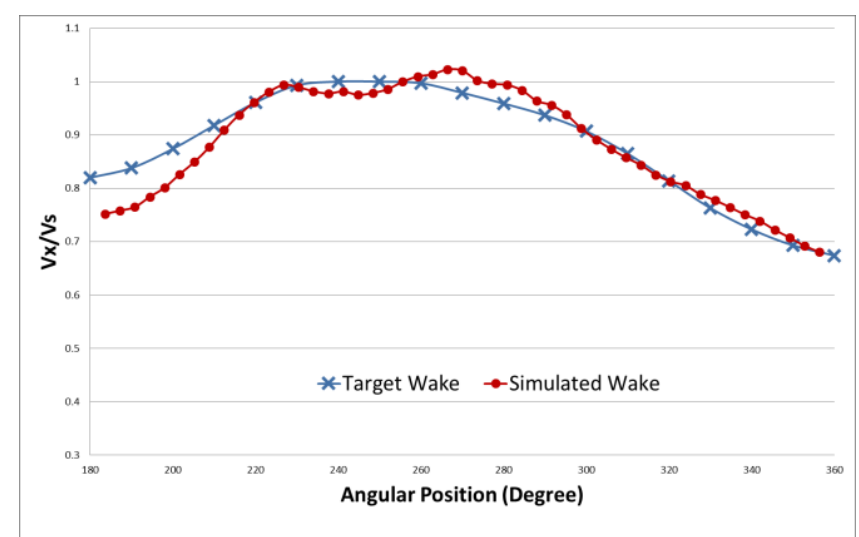

Figure 5 : Comparative plots of axial wake velocities at $\mathrm{r} / \mathrm{R}=0.827$ 


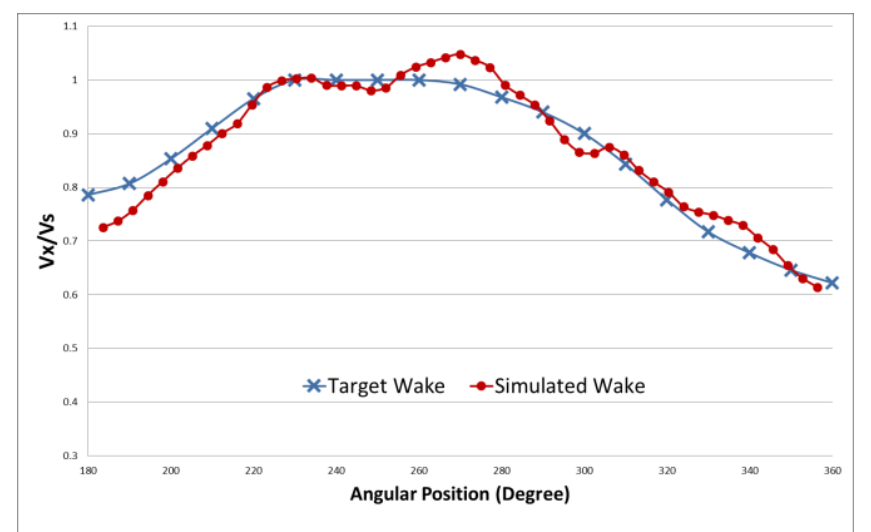

\begin{tabular}{|c|c|c|c|c|}
\hline DATA / PARTICULARS & \multicolumn{4}{|c|}{ VALUES } \\
\hline Power rating ( $\%$ of $\mathrm{MCR})$ & $\begin{array}{c}\text { Service } \\
\text { Condition at } \\
2.23 \% \mathrm{MCR} \\
\end{array}$ & $\begin{array}{c}\text { Service } \\
\text { Condition at } \\
6.9 \% \mathrm{MCR} \\
\end{array}$ & $\begin{array}{c}\text { Service } \\
\text { Condition at } \\
16.1 \% \mathrm{MCR} \\
\end{array}$ & $\begin{array}{c}\text { Service } \\
\text { Condition at } \\
73.4 \% \mathrm{MCR} \\
\end{array}$ \\
\hline Dynamic draught $\mathrm{T}_{\mathrm{A}} / \mathrm{T}_{\mathrm{F}}(\mathrm{m})$ & $1.95 \mathrm{~m} / 1.57 \mathrm{~m}$ & $1.95 \mathrm{~m} / 1.57 \mathrm{~m}$ & $1.95 \mathrm{~m} / 1.57 \mathrm{~m}$ & $1.95 \mathrm{~m} / 1.57 \mathrm{~m}$ \\
\hline Ship speed through water, $\mathrm{V}_{\mathrm{S}}(\mathrm{knot})$ & 4.775 & 7.100 & 9.350 & 15.108 \\
\hline $\begin{array}{l}\text { Engine speed, N (RPM) } \\
\text { (As set on the wheelhouse by the skipper) }\end{array}$ & 600 & 900 & 1200 & 2000 \\
\hline $\begin{array}{c}\text { Delivered power at propeller, } \mathrm{P}_{\mathrm{D}}(\mathrm{kW}) \\
\text { (Port side in full Scale) }\end{array}$ & 10.0 & 31.0 & 72.25 & 329.5 \\
\hline $\begin{array}{l}\text { Propeller speed, actual } \mathrm{N}_{\text {act }}(\mathrm{RPM})- \\
\text { (Port side in full Scale) }\end{array}$ & 342.8 & 514.2 & 682.1 & 1141.5 \\
\hline Cavitation number, $\sigma_{\mathrm{n}}$ & 1.20 & 0.53 & 0.30 & 0.11 \\
\hline $\begin{array}{c}\text { Torque }(\mathrm{kNm})- \\
\text { (Port side in full scale) }\end{array}$ & 0.3 & 0.6 & 1.0 & 2.8 \\
\hline Torque Coefficient, $10 \mathrm{~K}_{\mathrm{Q}}$ & 0.378 & 0.336 & 0.318 & 0.318 \\
\hline
\end{tabular}

Figure 6 : Comparative plots of axial wake velocities at $\mathrm{r} / \mathrm{R}=1.013$

\section{Cavitation test conditions}

The trial runs simulated in the tunnel were determined based on the four most representative and reliable runs of the fullscale trials selected by the project partners. These four conditions had sufficient repetitions and reciprocal runs to filter out the tidal current effects and included one non-cavitating condition and three cavitating conditions corresponding to the following engine speeds: 600, 900, 1200 and $2000 \mathrm{rpm}$. The corresponding propeller shaft speeds were lower, in the gearbox ratio of 1.75:1. A summary of the selected full-scale conditions and relevant data for the vessel is given in Table $\mathbf{3}$.

Table 3 : Selected full-scale test conditions and relevant data for "The Princess Royal" 
1 For the tunnel tests similarity was achieved in the following key parameters for each operational condition:

$$
J=\frac{V_{A}}{n D} \quad K_{Q}=\frac{Q}{\rho n^{2} D^{5}} \quad \sigma_{n}=\frac{P_{a}+\rho g h_{s}-P_{v}}{0.5 \rho(\pi n D)^{2}}
$$

Table 4 : A summary of cavitation tunnel test conditions

\begin{tabular}{|c|c|c|c|c|}
\hline DATA / PARTICULARS & \multicolumn{4}{|c|}{ VALUES } \\
\hline Power rating $(\%$ of MCR) & $\begin{array}{c}\text { Service } \\
\text { Condition at } \\
2.23 \% \mathrm{MCR}\end{array}$ & $\begin{array}{c}\text { Service } \\
\text { Condition at } \\
6.9 \% \mathrm{MCR}\end{array}$ & $\begin{array}{c}\text { Service } \\
\text { Condition at } \\
16.1 \% \mathrm{MCR}\end{array}$ & $\begin{array}{c}\text { Service } \\
\text { Condition at } \\
73.4 \% \mathrm{MCR}\end{array}$ \\
\hline Test conditions & Condition 1 & Condition 2 & Condition 3 & Condition 4 \\
\hline Cavitation number, $\sigma_{\mathrm{n}}(\pi \mathrm{nD})$ & 1.2 & 0.54 & 0.3 & 0.11 \\
\hline Dynamometer, $\mathrm{n}_{\mathrm{m}}(\mathrm{rps})$ & 15 & 20 & 20 & 30 \\
\hline Vacuum applied to tunnel, $\mathrm{H}_{\mathrm{st}}(\mathrm{mmHg})$ & -254 & -351 & -510 & -551 \\
\hline Model scale torque, $\mathrm{Q}(\mathrm{Nm})$ & 3.84 & 6.07 & 5.75 & 12.92 \\
\hline Adjusted inflow speed, $\mathrm{V}_{\mathrm{m}}(\mathrm{m} / \mathrm{s})$ & 1.39 & 2.25 & 2.41 & 3.75 \\
\hline
\end{tabular}

In Table 4 the first column contains the test condition numbers: 1, 2, 3 and 4 corresponding to the selected trials conditions (i.e. engine speed of) 600, 900, 1200 and 2000rpm, respectively given in Table 3. The defined cavitation tunnel tests conditions of the research vessel cover a wide range of speeds. Therefore, although fixed rotational speed and fixed vacuum application method was employed, it was concluded that the best way to cover such a wide range was to alter the propeller shaft speed in compliance with the full-scale propeller shaft speed. This also enabled the better cavitation observation in test Condition 4 by the reduction of the vacuum applied by means of increasing the propeller speed. 
During the whole course of the testing campaign, the water quality of the facility was monitored for gas content, since it is well known to affect cavitation. The dissolved oxygen content of the tunnel was kept at $30 \%$ during the experiments as recommended by ITTC (ITTC, 1987, 2011).

\section{Noise measurements}

\subsection{Equipment and data analyses}

7 The noise measurements were made using the Bruel \& Kjaer (B\&K) PULSE Type 3023 data acquisition system with a 6/1 LAN interface and a B\&K Type 8103 miniature hydrophone which was located inside the cavitation tunnel and supported by a streamlined strut. The offset of the hydrophone location is given in Table $\mathbf{5}$.

Table 5 Offset of hydrophone location in tunnel

\begin{tabular}{|c|c|}
\hline $\begin{array}{c}\text { 8103 Hydrophone location relative to } \\
\text { Propeller Plane \& shaft line intersection }\end{array}$ \\
\hline $\mathrm{x}$ & $415 \mathrm{~mm}$ \\
\hline $\mathrm{y}$ & $455 \mathrm{~mm}$ \\
\hline $\mathrm{z}$ & $150 \mathrm{~mm}$ \\
\hline
\end{tabular}

12 The noise signals were post-processed by the PULSE lab-shop using CPB and FFT analyzers that were constructed in the dedicated PULSE software. During post-processing, the acoustic data derived in $1 / 3$ octave bandwidth sound source level in $\mathrm{dB}$ relative to $1 \mu \mathrm{Pa}$. The frequency bandwidth ranged from $20 \mathrm{~Hz}$ up to $20 \mathrm{kHz}$ and the measurements were averaged exponentially over 1-second intervals to ensure satisfactory overlapping of the measurements whilst no acoustical weighting was applied.

The noise data acquisition was conducted by using the waterfall format of the PULSE software in order to eliminate the effect of any instantaneous sources. This was achieved by using the multi-buffer option of the software and by triggering the system every 0.25 seconds for the next measurement. The measurements were recorded for 50 triggers or 12.5 seconds at 25 $\mathrm{kHz}$ sampling rate. 
In order to ensure the repeatability and reliability of the measurements, Condition 4 was repeated three times since this was

2 the condition where the most severe cavitation was experienced and hence might have had a higher risk of errors. The uncertainty analysis for this condition has indicated a maximum error of $4.39 \mathrm{~dB}$ in the measured URN levels and is well within the range of the recommendations (1-5 dB) made by the ITTC (ITTC, 2014).

6 The noise data was acquired in raw format, which was subsequently corrected to an equivalent $1 \mathrm{~Hz}$ bandwidth and $1 \mathrm{~m}$ source level. A common practice in the analysis and presentation of the noise levels is to reduce the measured values of Sound Pressure Levels $(S P L)$ in each $1 / 3$ Octave band to an equivalent $1 \mathrm{~Hz}$ bandwidth by means of the correction formula recommended by ITTC (1987) as follows

$$
\mathrm{SPL}_{1}=\mathrm{SPL}_{m}-10 \log \Delta f
$$

where; $S P L_{1}$ is the Sound Pressure level in $1 \mathrm{~Hz}$ band in $\mathrm{dB}$ relative to $1 \mu P a ; S P L_{m}$ is the Sound Pressure level in $1 / 3$ Octave band in $\mathrm{dB}$ relative to $1 \mu \mathrm{Pa} ; \Delta f$ is the frequency bandwidth for the $1 / 3$ Octave band for each center frequency.

The ITTC also required that the sound pressure levels to be corrected to a standard measuring distance of $1 \mathrm{~m}$ using Equation 3 and based on the location of the hydrophone relative to the propeller as given in (ITTC, 1987)

$$
\mathrm{SPL}=\mathrm{SPL}_{1}+20 \log (r)
$$

where $S P L$ is the Sound Pressure level in $1 \mathrm{~Hz}$ band in $d B$ relative to $1 \mu P a$ at $1 \mathrm{~m} ; r$ is the distance of the location of the hydrophone distance from the propeller centerline.

Extrapolation of model test URN can be achieved using various scaling procedures to obtain the full-scale propeller noise levels (Bark \& Berlekom, 1978; Bark, 1985, 2000). However, various tunnel-related factors can result in erroneous acoustical cavitation testing leading to inaccurate noise prediction. Two such factors are the reverberant nature of the cavitation tunnel and the high level of background noise due to the tunnel impeller and model propeller drive systems etc.

These cause difficulties in interpreting the genuine propeller noise, as do other factors such as dissolved gas content, 
viscosity, etc. (ITTC, 1987). Therefore, accurate prediction of the full-scale propeller noise from model tests in a cavitation tunnel is not possible without a detailed knowledge of the influence of the proximity of the tunnel walls and other factors, affecting the measured noise. On the other hand, the determination of correlation factors to be applied to model measurements would involve a large programme of model and full-scale tests. For this reason, such correlation factors do not exist for the Emerson Cavitation Tunnel. In the absence of such an in-depth scrutiny of the tunnel performance for noise, linear acoustical coefficients have been utilized for the extrapolation coefficients, as proposed by Strasberg (Strasberg, 1977).

However, an approximation to the full-scale noise levels was made using the scaling laws recommended by the Cavitation Committee of (ITTC 1987). These laws are concerned only with differences in dimensions and operating conditions of the model and full-scale propellers and take no account of the fact that the model measurements may have been made in a cavitation tunnel. Furthermore the scaling laws are based on Rayleigh's equation for the radial motion of a single spherical cavity which is assumed to be in an inviscid and incompressible fluid (Plesset \& Prosperetti, 1977; Plesset, 1949). Equation 4 was used in combination with the ratio of the power spectral density expressions to represent the difference in the sound pressure levels (SPL) in the model and full-scale as follows:

$$
\Delta L_{S}=\mathrm{SPL}_{S}-\mathrm{SPL}_{M}=20 \log \left[\left(\frac{D_{S}}{D_{M}}\right)^{z}\left(\frac{r_{M}}{r_{S}}\right)^{x}\left(\frac{\sigma_{S}}{\sigma_{M}}\right)^{y / 2}\left(\frac{n_{S} D_{S}}{n_{M} D_{M}}\right)^{y}\left(\frac{D_{S}}{D_{M}}\right)^{y / 2}\right] d B
$$

where $\Delta L$ is the difference in the sound pressure levels; subscripts $S$ and $M$ refer to the ship and model respectively; $D$ is the propeller diameter which is equal to $0.75 \mathrm{~m}$ for the ship and $0.214 \mathrm{~m}$ for the model; $r$ is the reference distance for which the noise level is predicted and is equal to $1 \mathrm{~m}$ for both ship and model; $\sigma$ is the rotational cavitation number which has the same value for ship and model; $n$ is the propeller rate of rotation; and $\rho$ is the mass density of water which has a measured value of $1002 \mathrm{~kg} / \mathrm{m}^{3}$ for the cavitation tunnel and the standard value of $1025.9 \mathrm{~kg} / \mathrm{m}^{3}$ for the sea water. With these values and inserting $\mathrm{x}=1, y=2$ and $z=1$, the scaling of the noise levels and frequency shifts were estimated for all the conditions specified. 
The frequency shift from model to full-scale is based on the collapse time of a single bubble as given in Equation 5:

$$
\frac{f_{P}}{f_{M}}=\frac{n_{P}}{n_{M}}
$$

2

where $f_{P}$ is the model scale frequency associated with full scale frequency $f_{M}$.

One important practical issue in the analysis of the data is associated with the catamaran configuration of the target vessel, which has two propellers and hence two dominant noise sources. However, in the tunnel tests only one of these sources was represented by the use of the starboard demi-hull. The missing propeller effect was accounted for by doubling the measured levels. This was achieved in the logarithmic scale by adding $3 \mathrm{~dB}$ to the measured levels.

\subsection{Presentation of results}

Figure 7 to Figure 10 present comparisons of the total Radiated Noise Levels (RNL), measured in the full-scale trials by Brooker \& Humphrey (2014), with the total RNL extrapolated based on Newcastle's (UNEW) tunnel measurements for each of the four operating conditions given in Table 4, respectively. The full-scale trials are conducted for each condition given within the Table 3 with 2 double runs. The double runs include a Northwards and Southwards course headings for both starboard and port aspect. The trial area depth was chosen to be approximately $100 \mathrm{~m}$ with soft mud seabed type. The measurements are made using a hydrophone array with 3 hydrophones with the maximum depth of the deepest hydrophone being $50 \mathrm{~m}$. The full-scale measurements are analysed using the dipole source level assumption which is also referred as the affected source level or RNL. Whilst the RNL representation does not take into account for the influence of the well-known Lloyd's Mirror effect, the analysis procedure adopted using ANSI standard minimizes the error introduced by the averaging applied to the measurements made with three hydrophones.

Figure 7 to Figure 10 also include the measured background noise levels in both the full-scale trials and cavitation tunnel experiments. Although the nature of the background noise for the model and full-scale noise measurement is different, it is interesting and important to note the level of contribution from these sources. Whilst there is a significant contribution from the background in the tunnel measurements, such contribution in the full-scale measurements from the ambient noise is rather small. Further information on the definition of the background noise levels for the full-scale trials and model tests is given in the following part of this section. 


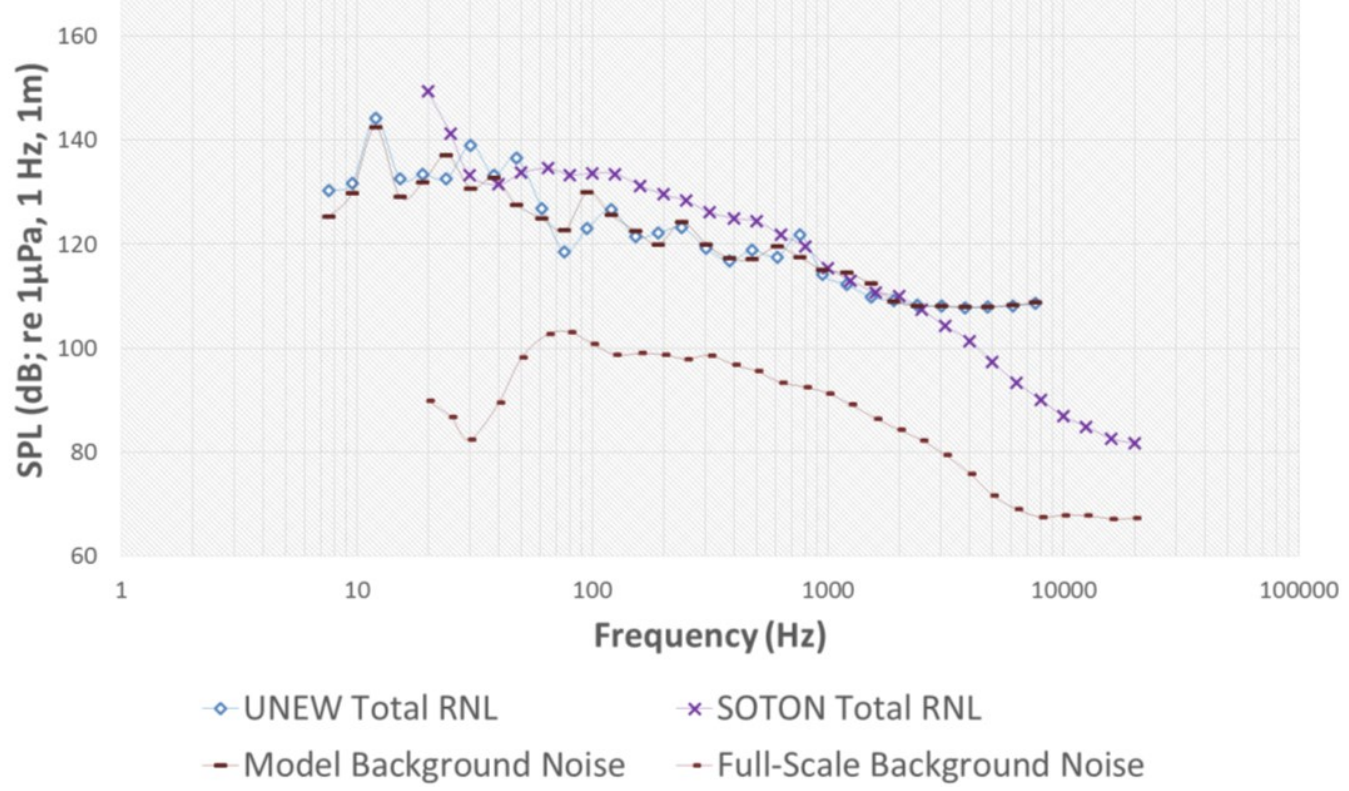

Figure 7 : Condition 1 - Comparison of total radiated noise levels from full-scale trials and tunnel test measurements based extrapolations

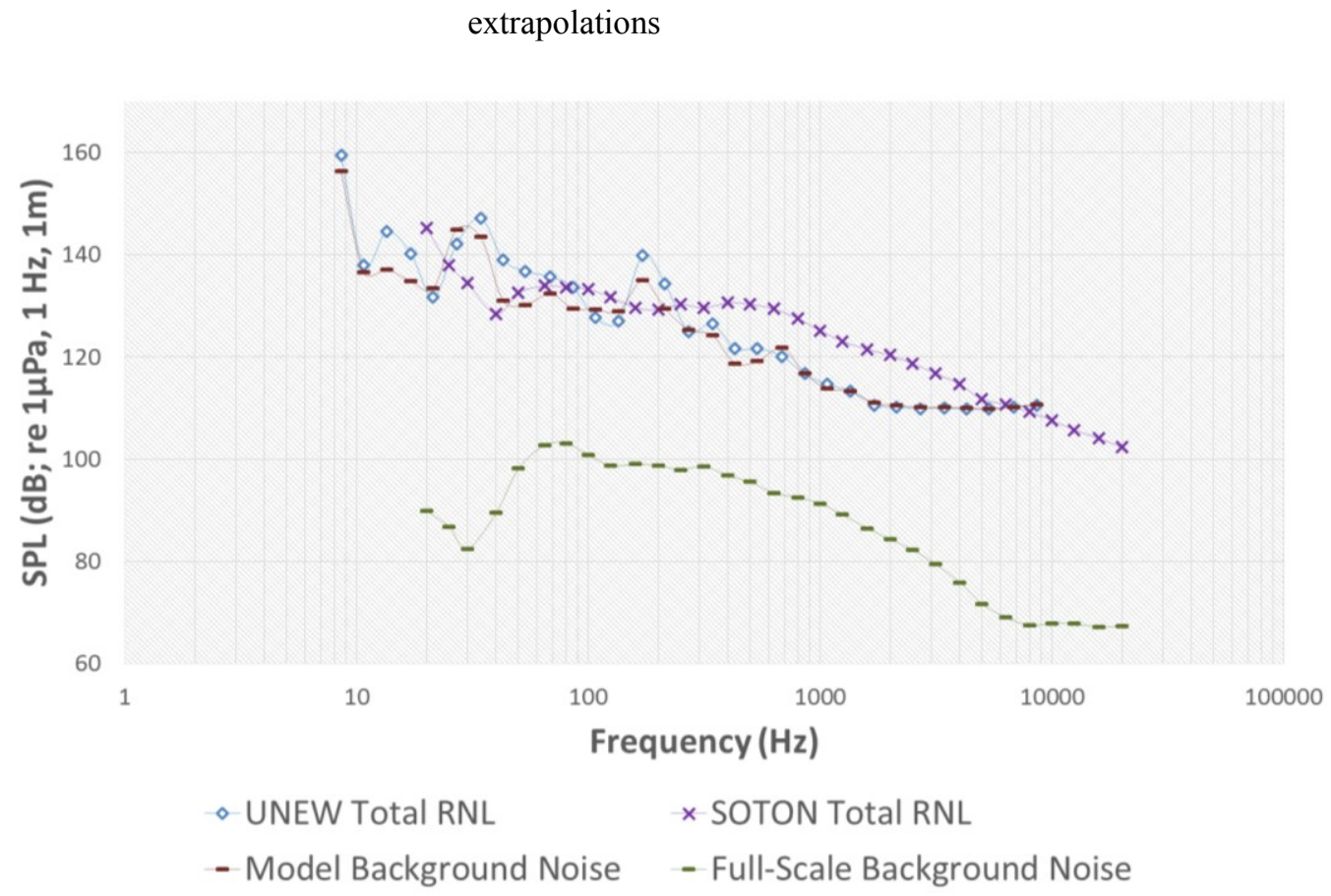

Figure 8 : Condition 2 - Comparison of total radiated noise levels from full-scale trials and tunnel test measurements based extrapolations 


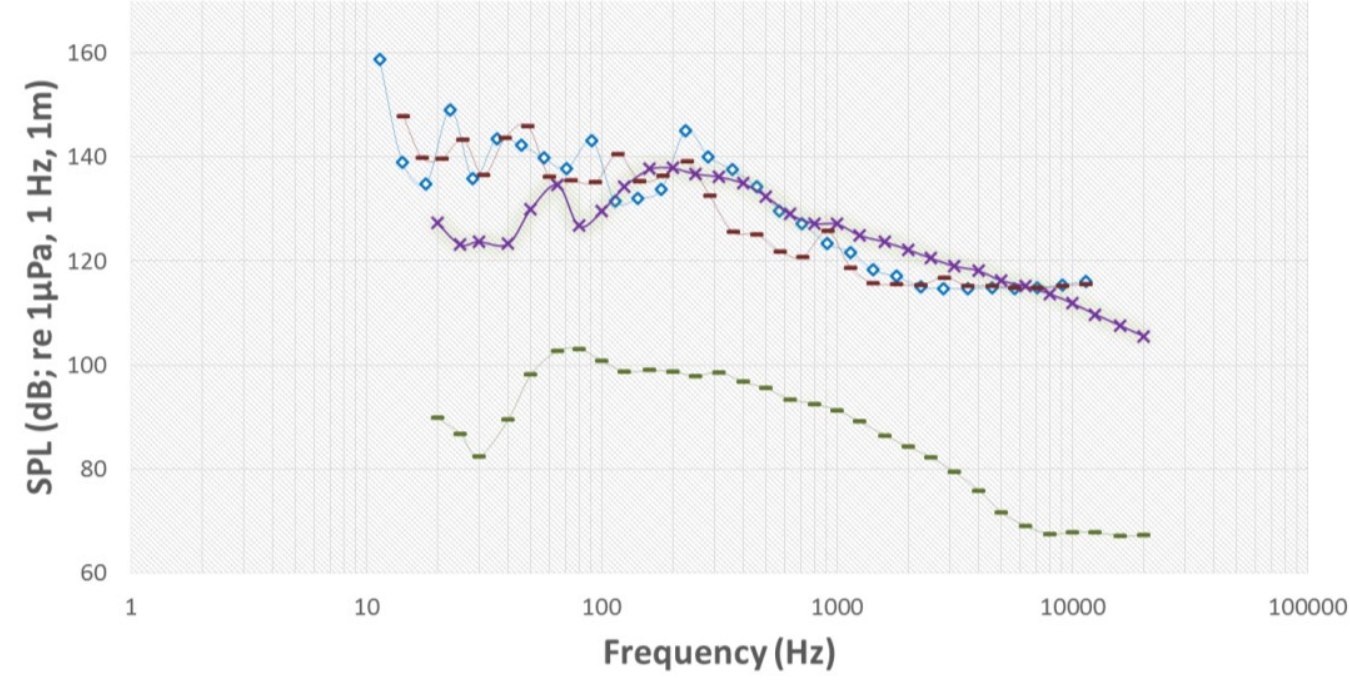

- UNEW Total RNL

* SOTON Total RNL

- Model Background Noise

- Full-Scale Background Noise

Figure 9 : Condition 3 - Comparison of total radiated noise levels from full-scale trials and tunnel test measurements based extrapolations

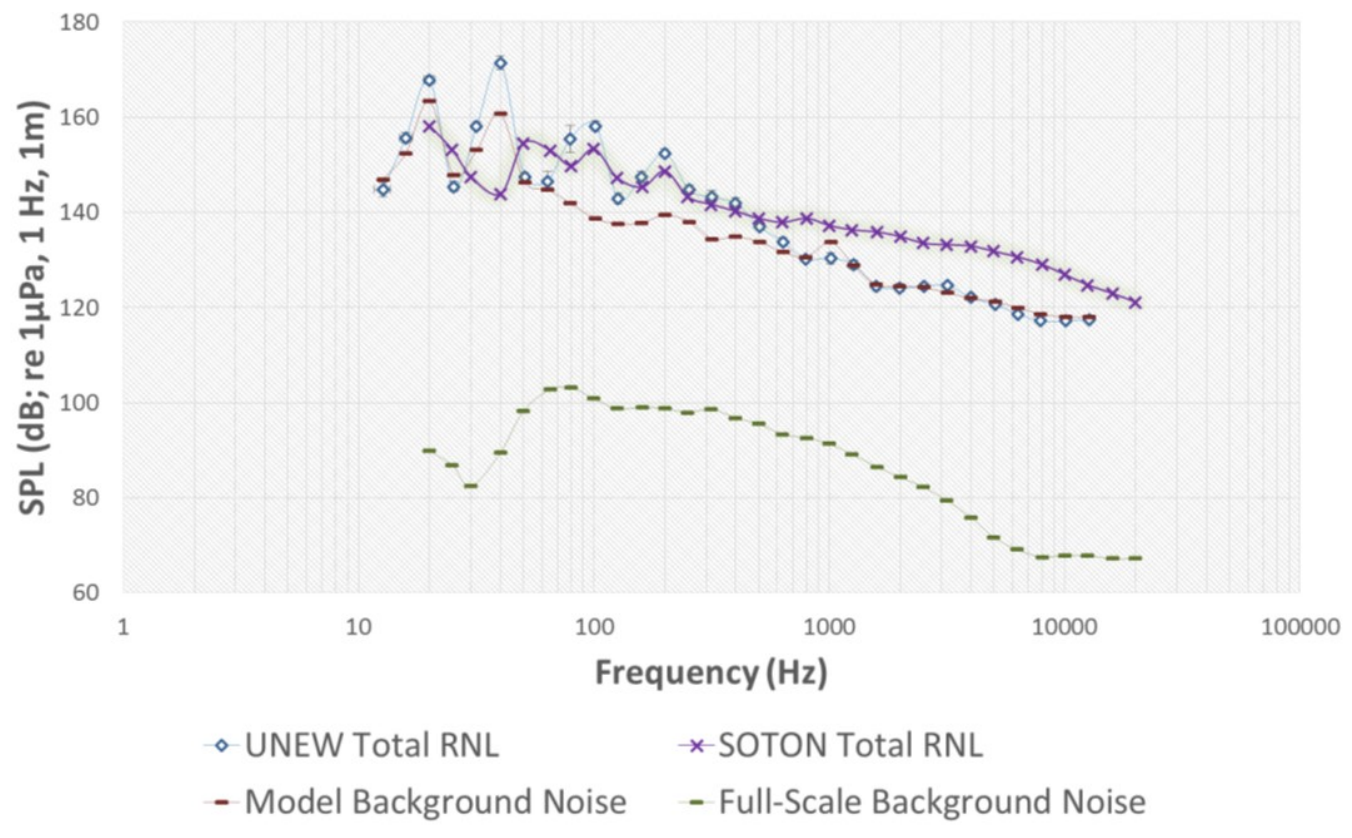

Figure 10 : Condition 4 - Comparison of total radiated noise levels from full-scale trials and tunnel test measurements based extrapolations 
In Figure 11 through 14 the comparative "net" RNLs are presented for the same four operating conditions after making corrections for the background noise measurements following the recommendations given by ITTC (ITTC, 2014) and ANSI (ANSI, 2009).

For the tunnel background noise measurements a series of tests was conducted for the corresponding operating conditions given in Table 4 without the model propeller which was replaced by a dummy hub (ITTC, 2014). If the difference in the measured noise levels with the propeller and background noise is too small, the measurements need to be corrected following the procedure in ANSI/ASA S12.64-2009/Part1. When the difference is smaller than $3 \mathrm{~dB}$, the result is discarded. In case of a difference between 3 and $10 \mathrm{~dB}$, the results are corrected according to Equation 7 and no correction is applied in case of the difference being greater than $10 \mathrm{~dB}$. The influence of the background correction on different conditions are scrutinized in detail in section 4.3 .

$$
\left.\left.S P L_{N}=10 \log \left[10^{\left(S P L_{T} / 10\right.}\right)-10^{\left(S P L_{B} / 10\right.}\right)\right]
$$

where subscripts $N, T$ and $B$ indicate net, total and background respectively.

Within the scope of the background noise level assessment of the tunnel tests, the dummy model approach necessitated the use of the wake screen to be located in a transverse plane at a distance of 1.5 propeller diameter upstream of the propeller's hub center. This may raise concerns over the noise creating mechanisms introduced to the experiment by the presence of the wake screen such as cavitation, increase in turbulence intensity and singing. The effects of these sources on the measured noise levels were considered to be taken care of through the background noise correction.

Although the ITTC guidelines recommend further correction of the measured data to account for the influence of the testing environment such as the reverberation and reflections due to the walls (ITTC, 2014), such corrections were not applied to the presented results. This was purely due to the time restrictions imposed on the project and tight tunnel schedule that was not able to accommodate a detailed investigation for this dummy model.

The noise measurements in Figure 15 through 18 are supported by images of the cavitation observations both from the tunnel tests and from full-scale trials for each of the four operating conditions, respectively. The qualitative information provided by the cavitation images provides further insight into the trends shown by the spectral levels as discussed in the next section. 


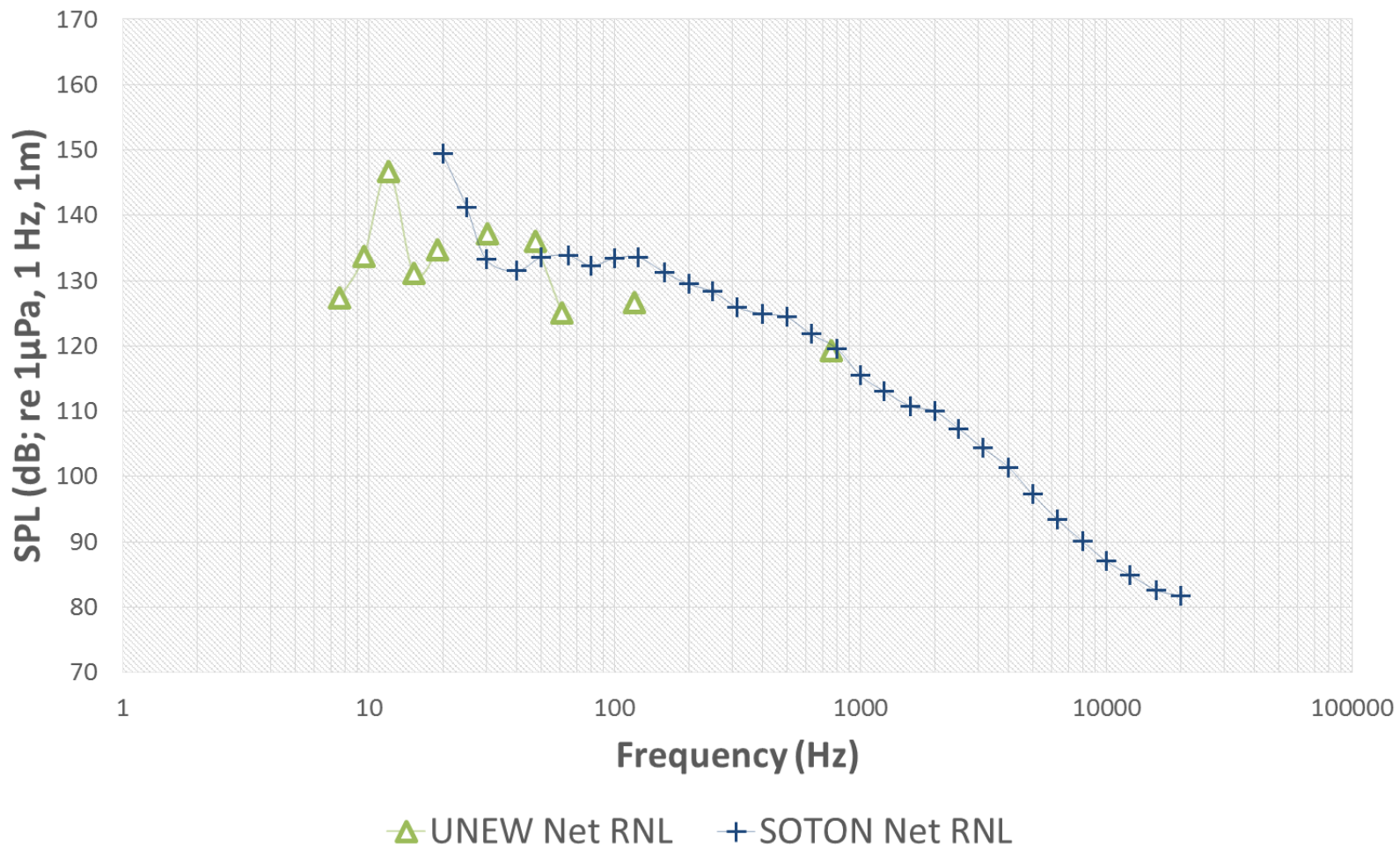

Figure 11 : Condition 1 - Comparison of net radiated noise levels from full-Scale trials and tunnel test measurement based on extrapolations.

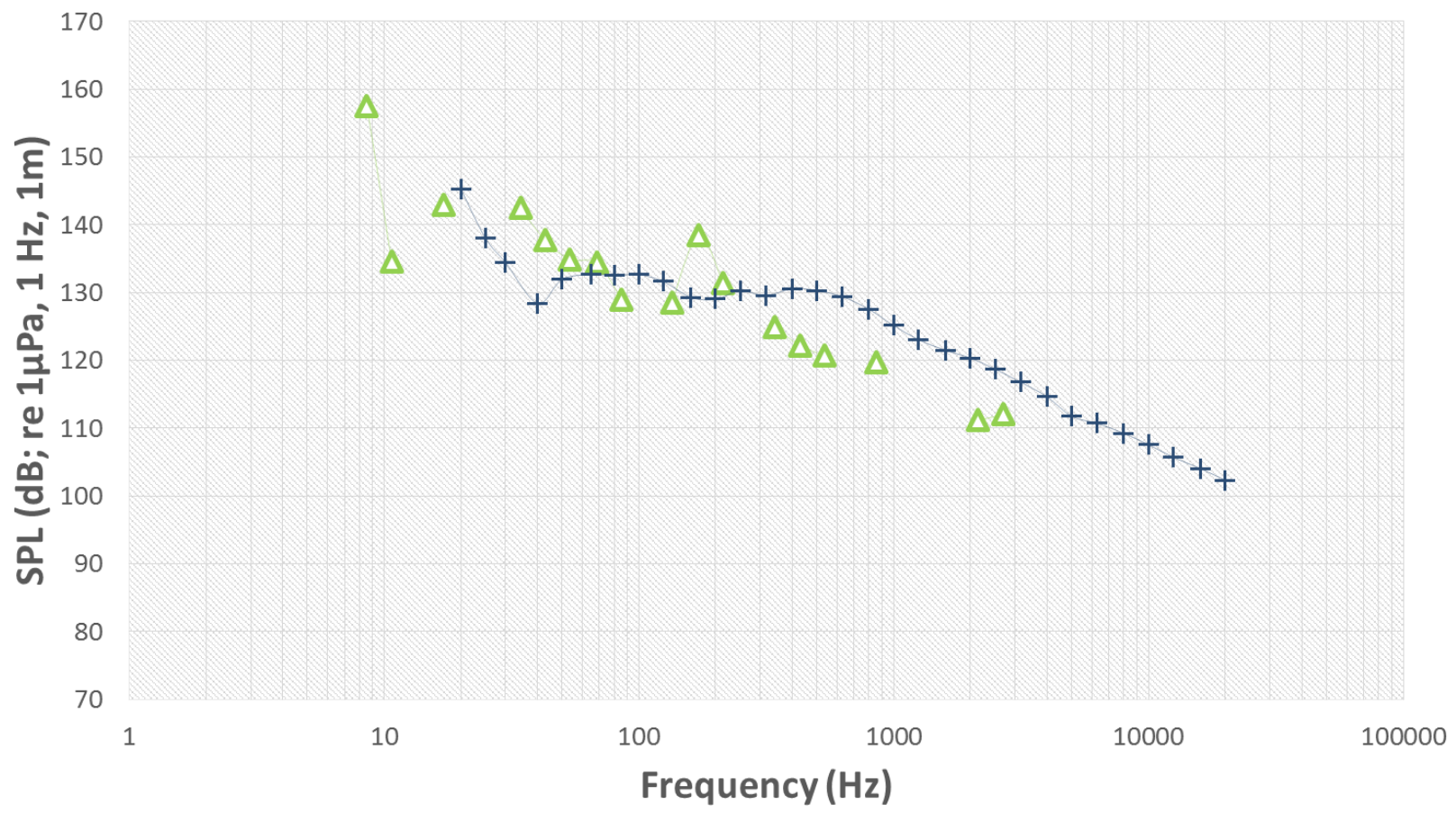

$\triangle$ UNEW Net RNL $\quad+$ SOTON Net RNL

Figure 12 : Condition 2 - Comparison of net radiated noise levels from full-scale trials and tunnel test measurement based on extrapolations 


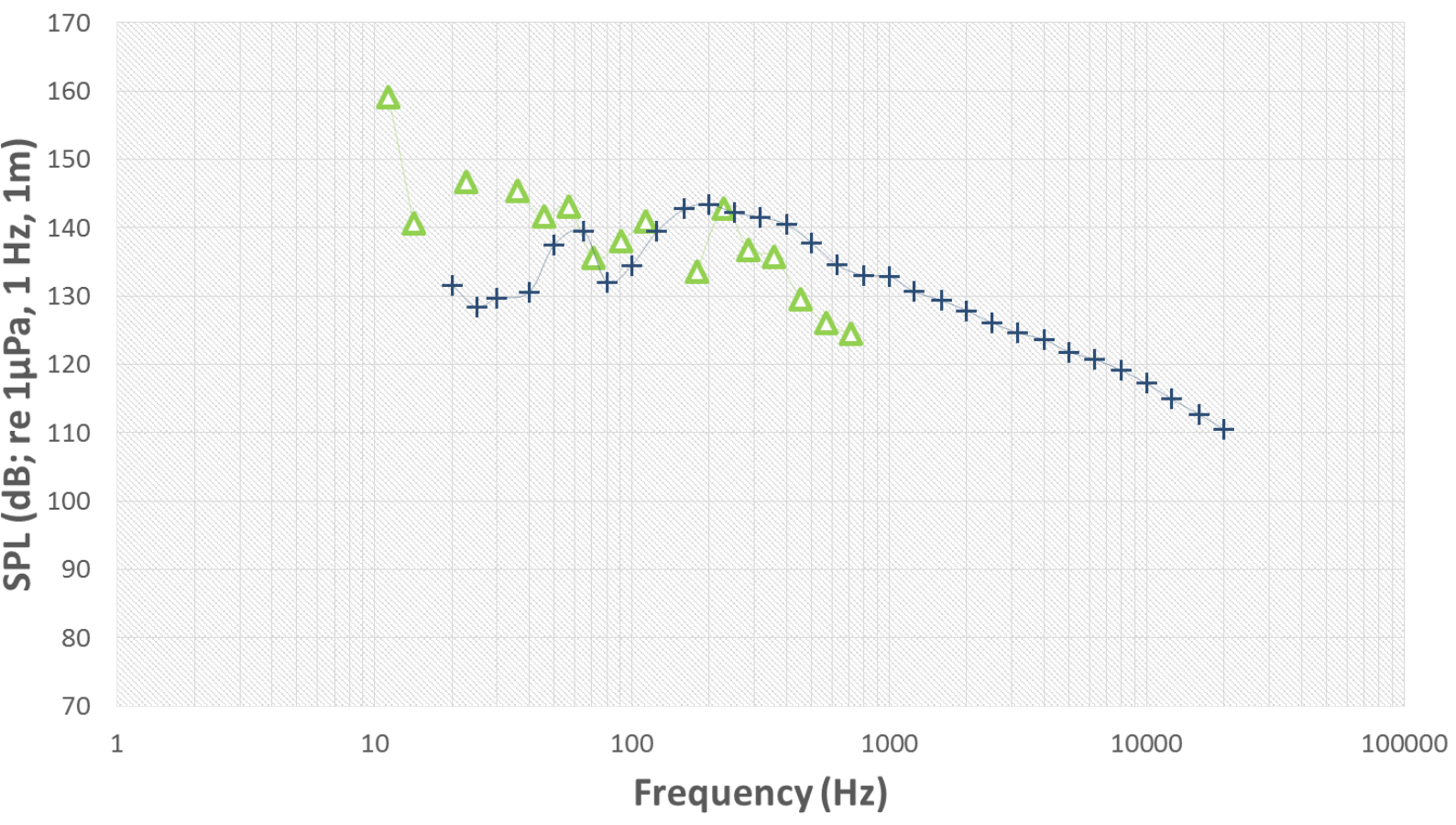

\section{$\triangle$ UNEW Net RNL $\quad+$ SOTON Net RNL}

Figure 13 : Condition 3 - Comparison of radiated noise levels from full-Scale trials and tunnel test measurement based on extrapolations

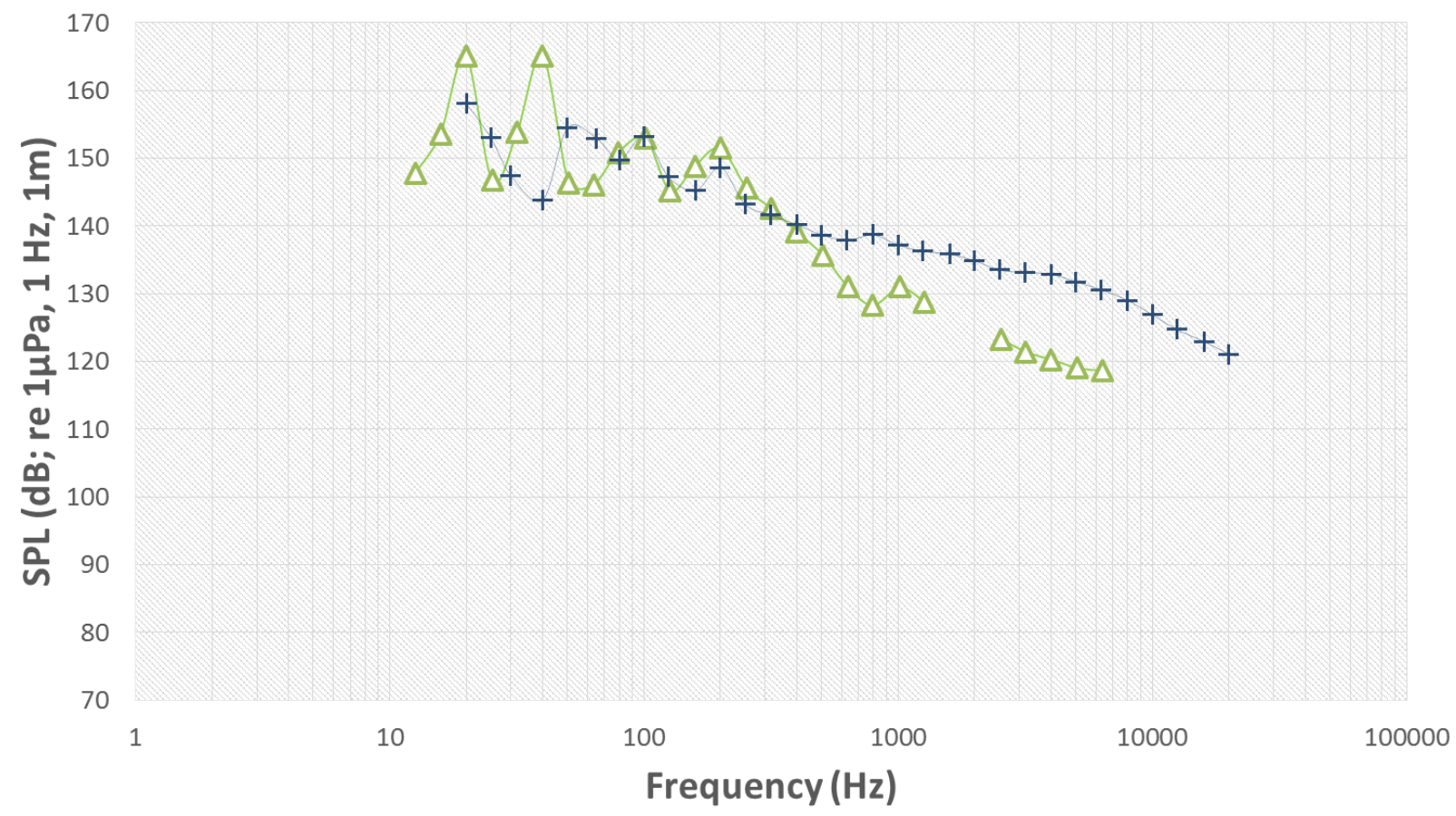

$\triangle$ Unew Net RNL + + SOTON Net RNL

Figure 14 : Condition 4 - Comparison of net radiated noise levels from full-scale trials and tunnel test measurement based on extrapolations 


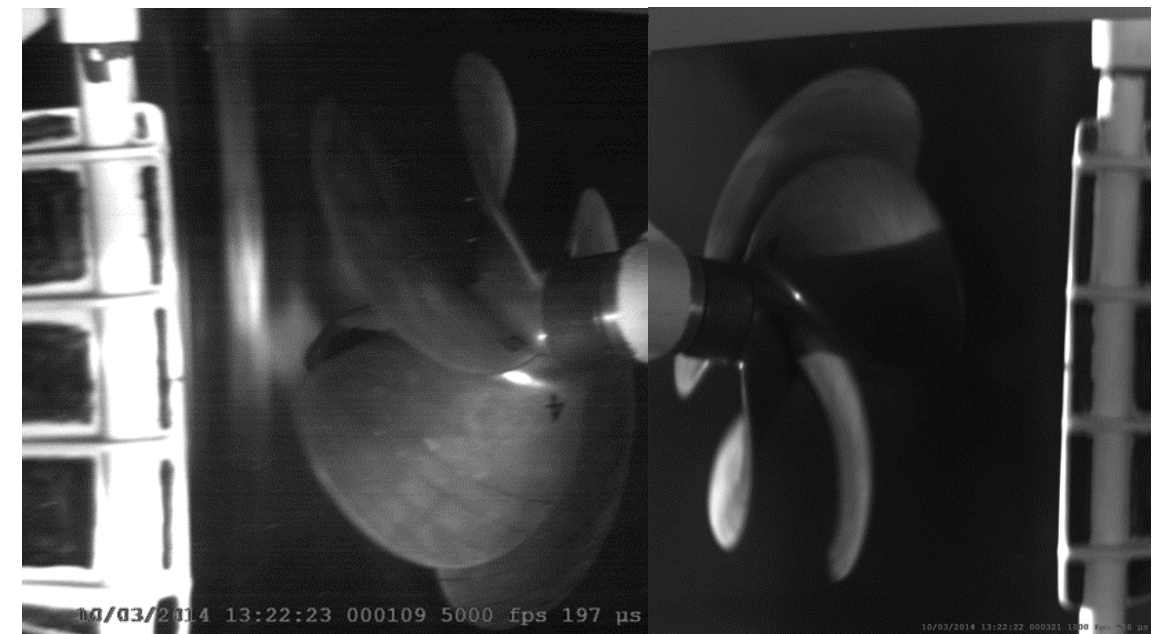

Figure 15a : Cavitation observations from tunnel tests for Condition 1

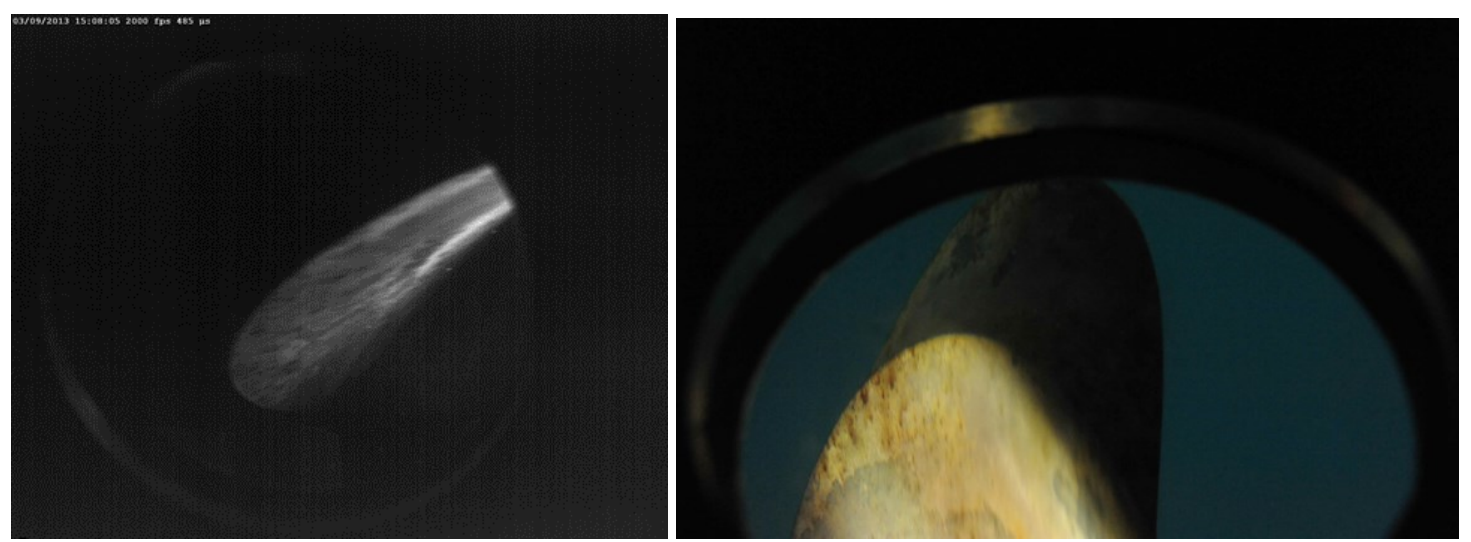

Figure 15b : Cavitation observations from full scale trials for Condition 1

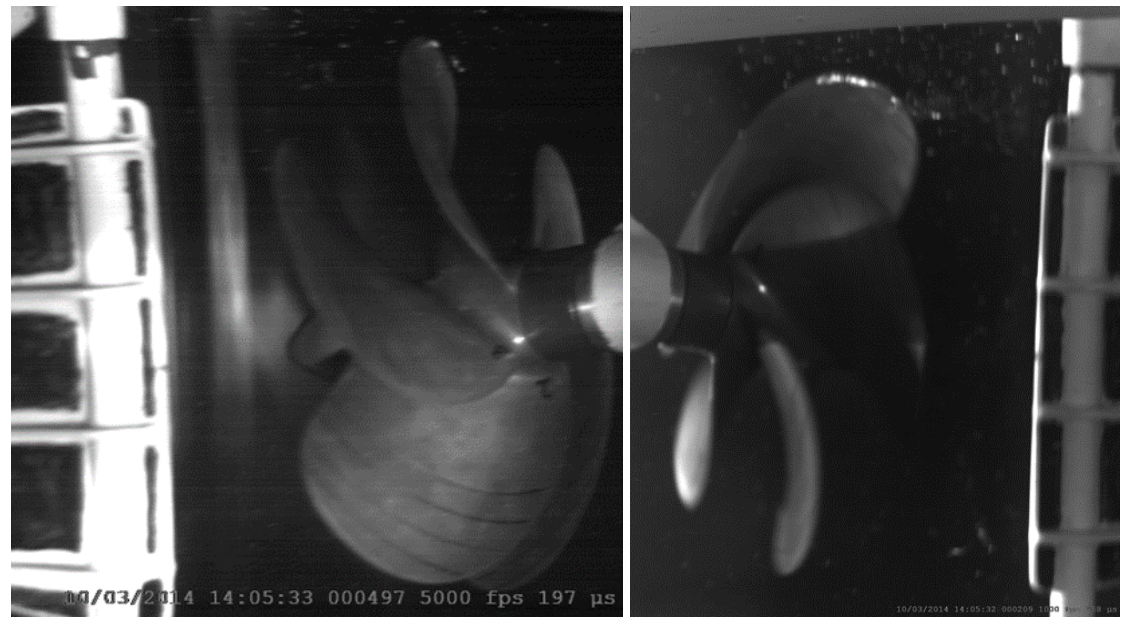

Figure 16a : Cavitation observations from tunnel tests trials for Condition 2 


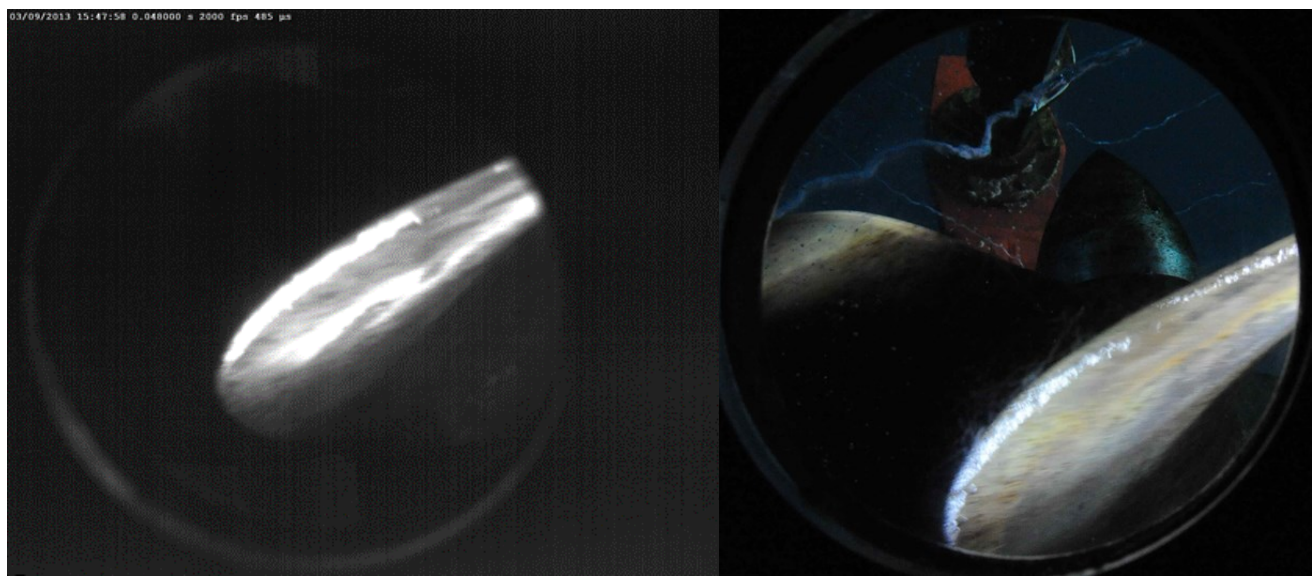

Figure 16b : Cavitation observations from full scale trials for Condition 2

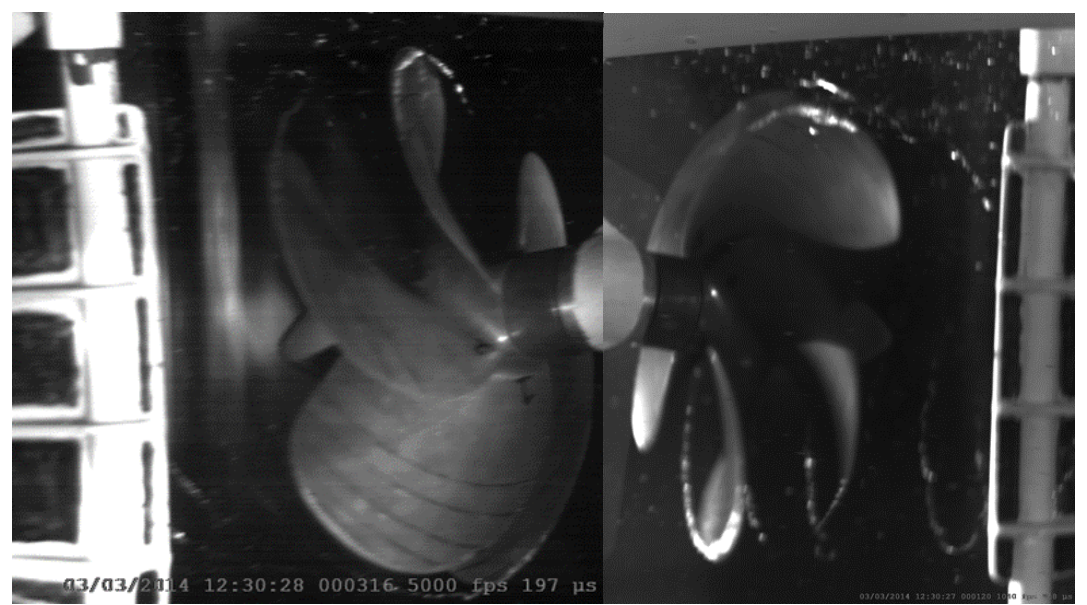

Figure 17a : Cavitation observations from tunnel tests for Condition 3

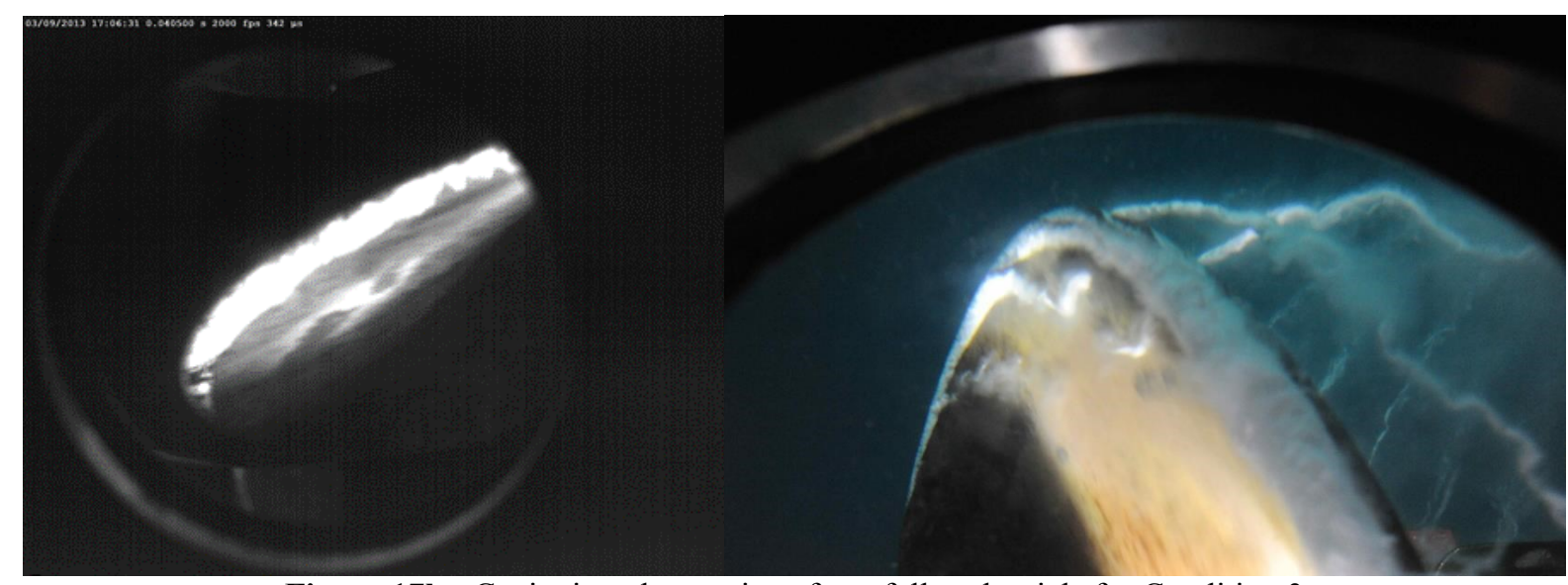

Figure 17b : Cavitation observations from full scale trials for Condition 3 

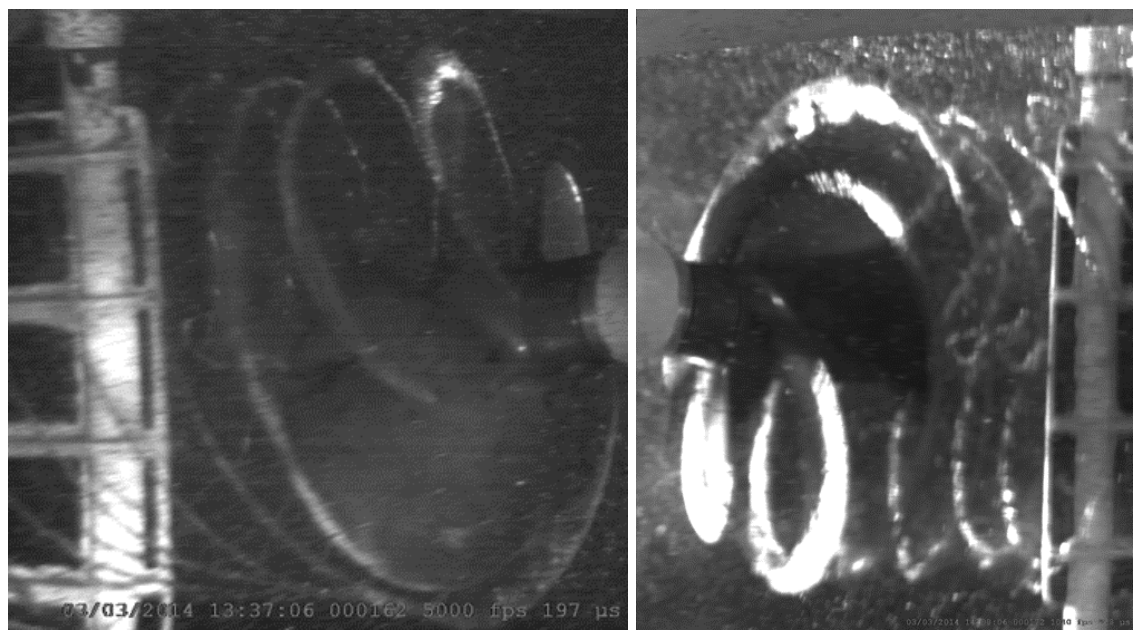

Figure 18a : Cavitation observations from tunnel tests for Condition 4

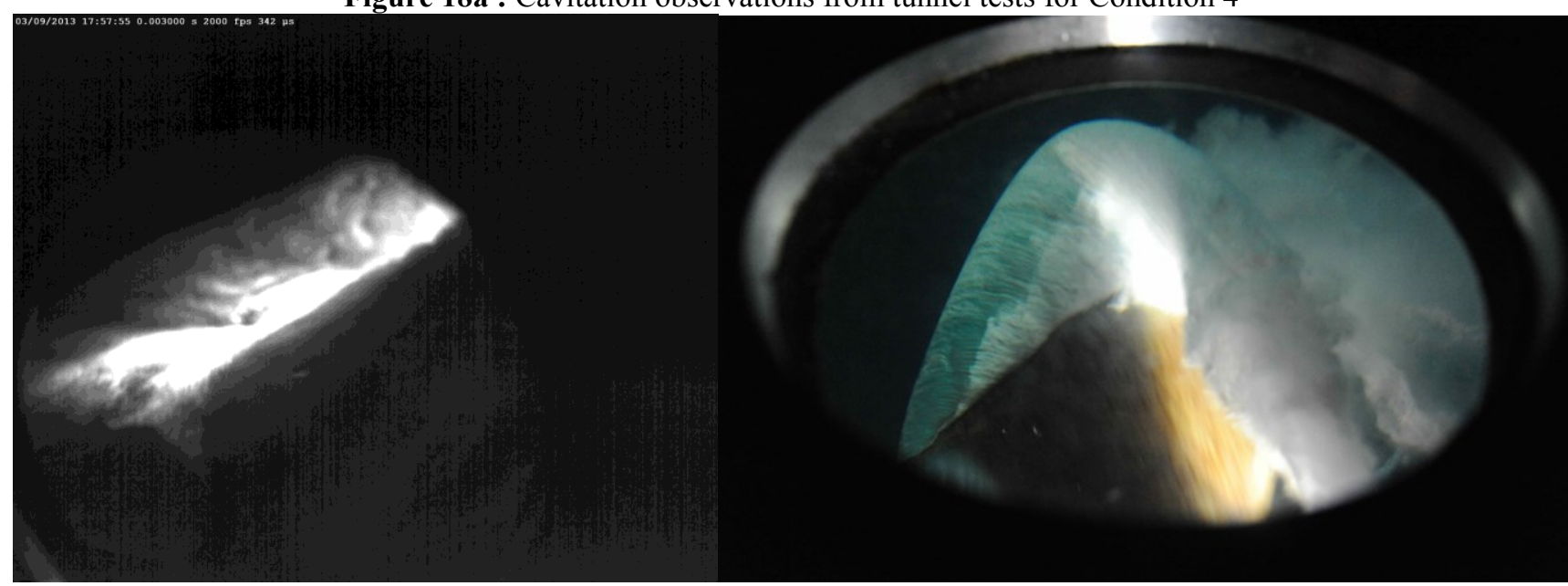

Figure 18b : Cavitation observations from full scale trials for Condition 4

\subsection{Discussion}

As shown in Figure 7 to Figure 10 the presentation of the total noise levels with the background noise measurements provides further insight especially in the tunnel measurements for which the background noise levels are much more significant. The background noise levels are even observed to exceed the total noise levels at certain frequencies where various dominant noise sources present in the tunnel tests are the major sources. This consequently means that for this certain case, the measured levels higher than the $1 \mathrm{kHz}$ are significantly influenced by the tunnel background noise sources.

When the background noise corrections were applied, as shown in Figure 11, for "Condition 1" most of the noise spectrum had to be discarded since the differences in the noise levels between the measured data with the propeller and background noise were less than $3 \mathrm{~dB}$. The noise spectrum for this non-cavitating condition was of the dominated by the background noise. One may thus consider if the application of Equation 4 and 5, which are based on cavitating bubble dynamics, is 
appropriate for this condition. Nevertheless, no further investigation was made in this study of non-cavitating noise extrapolation laws, since the focus was on the cavitation induced noise.

3 In Conditions 2 and 3, as shown in Figure 12 and Figure 13 respectively, the spectral levels are observed to be less affected 4 by the tunnel background noise for frequencies lower than $1000 \mathrm{~Hz}$. However, beyond this threshold, the spectral levels for 5 both conditions are still affected significantly by the background noise. The cavitation patterns for Conditions 2 and 3 , as shown in Figure $16(\mathbf{a}, \mathbf{b})$ and Figure $17(\mathbf{a}, \mathbf{b})$ respectively, indicate that the developed leading edge tip vortex and sheet cavitation is extremely unsteady, breaking-up (and bursting) intermittently with a cloudy appearance. The sheet cavitation terminates at the blade tip region by rolling-up in the form of a rather thick, intense and cloudy tip vortex, trailing to the rudder. Comparison of both the tunnel cavitation observations and the URN predictions with the full-scale measurements for these two conditions seem to be in reasonable agreement up to $500 \mathrm{~Hz}$ for the noise predictions.

The under estimation over the frequencies higher than $500 \mathrm{~Hz}$ can be due to various noise emitting mechanisms and cavitation dynamics created by the tip vortex cavitation. In order to ensure similar dynamics in model scale experiments one may suggest to establish a relationship based on the acoustical and cavitation similarity as proposed by Latorre and Shen \& Strasberg (Latorre, 1982; Shen \& Strasberg, 2003) using McCormick's earlier work (McCormick, 1962) although this was not tried in this study. It was also noted that the cavitation dynamics for Condition 3 was more active in full-scale than in the model tests and hence resulting in a distinct difference between the cavitation tunnel prediction and the full-scale counterpart that were observed in the high-speed videos from both cavitation tunnel tests and full-scale trials.

For Condition 4, as shown in Figure 14, the high level of URN noise spectra over the entire frequency range appears not to be significantly affected by the background noise since there is significant sheet cavitation and a complex interaction with the tip vortex cavitation. Figure 18a and Figure 18b, show that the propeller developed a rather large extent of suction side cavitation (almost $25-30 \%$ of the blade area) with increasing volume and intensity. This was observed to be rather unstable foamy sheet cavitation. The unsteadiness was more towards the blade tip where the sheet cavity terminated on the blade by rolling-up and increasing the strength and thickness of trailing tip vortex extending to the rudder. The trailing vortex sometimes broke-up intermittently, having a cloudy appearance. Also noted were a continuous, intensified cavitating Hub Vortex and, intermittently, a cavitating Hull-Propeller Vortex. The predictions for this condition underestimate the full-scale measurements at the high frequency region $(500 \mathrm{~Hz}-20 \mathrm{kHz})$. Although the cavitation extent comparisons are in reasonable agreement, violent collapse mechanisms and partial break-off of the sheet cavitation, identified to be a major noise emitting 
1 mechanism (Bark, 1986), cannot be adequately represented in model scale tests and hence may influence the under 2 prediction of the noise levels for this condition in the high frequency region.

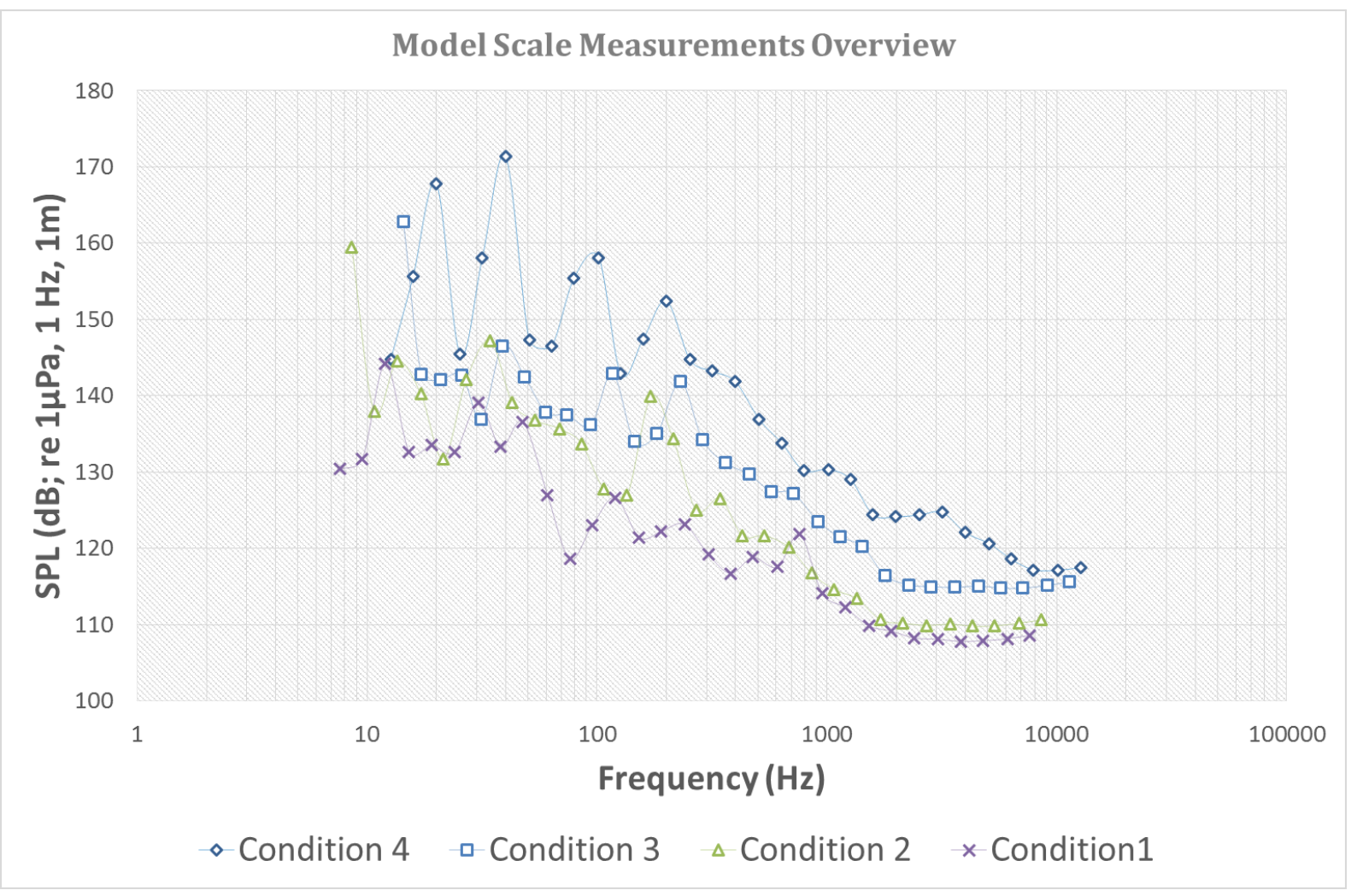

Figure 19: Model scale total noise measurements overview 


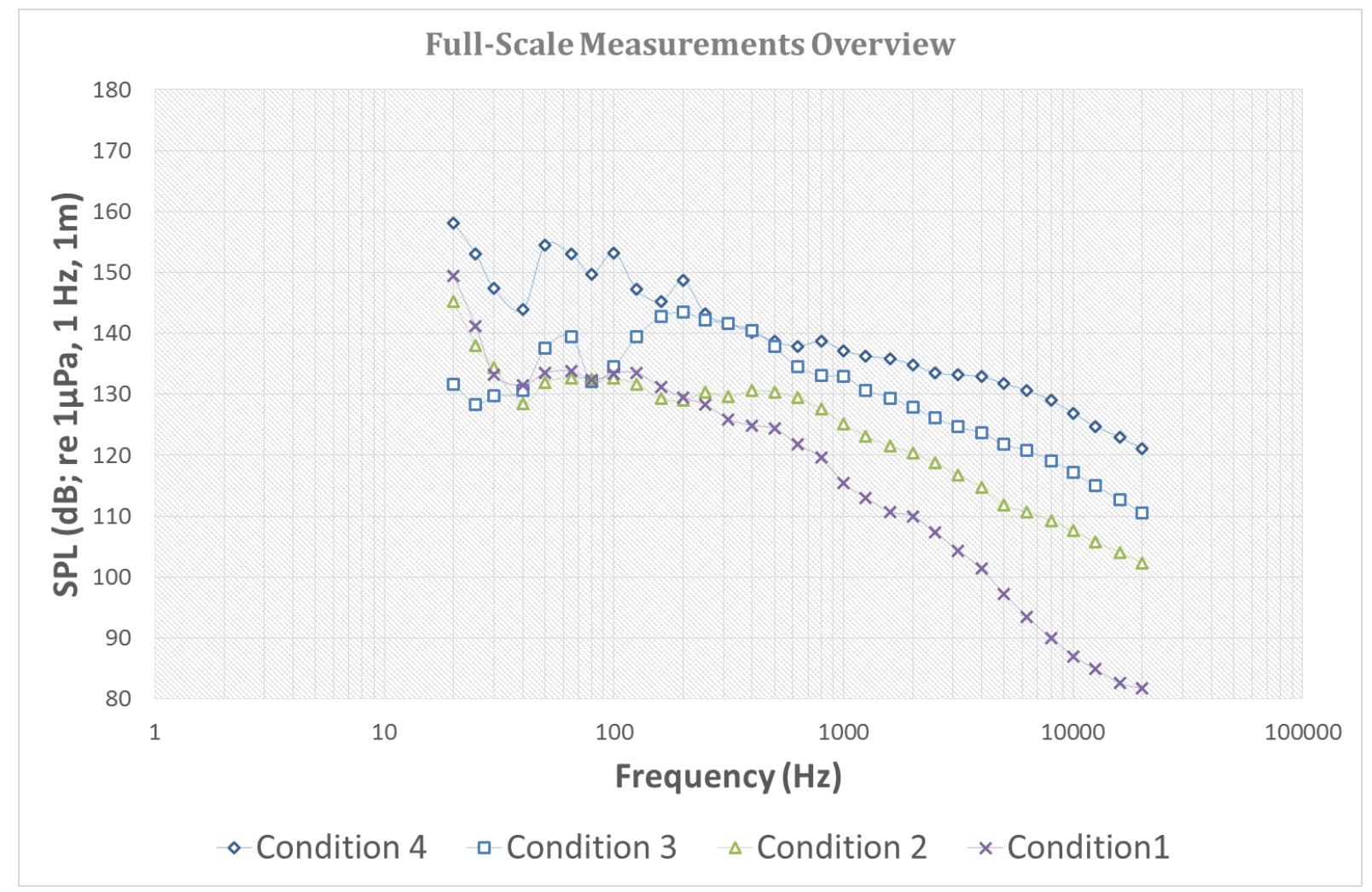

Figure 20 : Full-scale net noise measurements overview

Figure 19 and 20 give a comparative summary of the spectral levels for the four operating conditions at model and fullscale, respectively. These allow further interpretation of the impact of the operating conditions and associated cavitation dynamics on the noise spectra. It is clear from the both figures that whilst there is no cavitation, the URN levels are significantly low. However, as soon as cavitation inception occurs, the spectral levels increase. In Condition 2 the increase in the URN levels starts at a frequency around $200 \mathrm{~Hz}$ and is considered to reflect the relatively smaller diameter of the tip vortex cavitation and less activity in its cavitation dynamics. In Condition 3 the noise levels observed are to increase over the whole frequency range due the broadband nature of the cavitation phenomenon creating a direct impact especially in the high frequency range. The only test condition with the large extent of sheet cavitation is Condition 4, which shows a significant increase in the URN level over the whole frequency range. A series of peaks around multiples of the blade rates is connected both with increased volume variation of the sheet cavitation and with a larger diameter tip vortex cavitation with increased cavity dynamics.

In discussion of the results, it is appropriate to comment on the selected scale ratio (3.5) of the model tests. This is considerably smaller than typical scale ratios selected for testing other ship models, especially for representing large commercial ships. Although such a small ratio was imposed by the small size of the Princess Royal vessel relative to the 
medium size of the Emerson Cavitation Tunnel (ECT), other studies have been conducted by the Authors in the ECT using larger scales. One of these investigations involved the comparative URN predictions for a Fisheries Research Vessel (Atlar et. Al., 2001). In this experimental investigation, the full-scale URN measurements were compared with the predictions using a model propeller with a scale of 1:7 and behind a simulated wake using $2 \mathrm{D}$ wake screens. The comparisons of the predictions were in reasonable agreement with the full-scale measurements. In a very recent other investigation, Aktas et al. (2015) conducted predictions based on a small systematic series of model propellers tested in the ECT with a representative scale of 1:23 and behind systematically varied wake screens. The predictions were comparable with the average commercial shipping URN data given by Wales \& Heitmeyer (2002). In summary, the reasonable correlations of the URN predictions with the full-scale measurements obtained in the above mentioned two investigations (using relatively larger scale factors, i.e. 7 and 23 as opposed to 3.5 of the present study) should provide reasonable confidence for the range of scale ratios used in ECT for such tests.

\section{Conclusions}

This paper reports on experimental investigations to predict URN levels for the Newcastle University's catamaran research vessel "The Princess Royal". The findings are based on a series of model tests carried out in the university's medium size facility "The Emerson Cavitation Tunnel" and on full-scale noise trials conducted with the vessel as part of a collaborative European research project. Based on the investigations it can be concluded that

- In a medium size cavitation tunnel, a truncated dummy-hull model with properly scaled bow and stern sections, combined with the wake screens strategically fitted at the stern, could be the closest alternative to a full (twin-hull) model configuration to simulate the wake flow effectively.

- In spite of various simplifications made in the dummy-hull configuration to represent the actual catamaran vessel, the tunnel test measurements for underwater radiated noise levels and cavitation observations can provide a reasonable basis to validate the full-scale trial measurements by using the ITTC procedures and guidelines.

- Extrapolated URN spectra, based on tunnel tests in the presence of cavitation displayed more reasonable agreement with the full-scale URN over the low and medium frequency ranges than over the higher frequency range. 
- In the present study, the extrapolation issues related to the effect of the reverberations of the cavitation tunnel facility and non-cavitating operating conditions have not been tackled due to time restrictions on the project and a focus on the cavitation induced noise, and may require further investigations.

\section{Acknowledgments}

The research constituting the basis for this study has been supported by the European Union $7^{\text {th }}$ Framework Programme (FP7) under grant agreement No: 314394 through the SONIC project.

\section{References}

Abrahamsen, K. (2012). The ship as an underwater noise source. In Proceedings of Meetings on Acoustics, Acoustical Society of America (Vol. 17, p. 070058).

Aktas, B., Atlar, M., Fitzsimmons, P., Shi, W., Turkmen, S., \& Sasaki, N. (2015). Systematic cavitation tunnel tests for cavitation noise prediction of commercial ships using a standard series approach. In The 4th International Conference on Advanced Model Measurement Technologies for the Maritime Industry. Istanbul, Turkey.

Arveson, P., \& Vendittis, D. (2000). Radiated noise characteristics of a modern cargo ship. The Journal of the Acoustical Society of America, 107(1), 118-129.

Atlar, M. (2000). A history of the Emerson Cavitation Tunnel. International Conference on Propeller Cavitation, (NCT'50), Newcastle upon Tyne, UK, 3-5 April, 3-34.

Atlar, M. (2011). Recent upgrading of marine testing facilities at Newcastle University. In The 2nd International Conference on Advanced Model Measurement Technologies for the Maritime Industry (AMT'11). Newcastle upon Tyne, UK.

Atlar, M., Takinaci, A. C., Korkut, E., Sasaki, N., \& Aona, T. (2001). Cavitation Tunnel Tests for Propeller Noise of a FRV and Comparisonswith Full-Scale Measurements. International Symposium on Cavitation CAV2001.

Bark, G. (1985). Prediction of propeller cavitation noise from model tests and its comparison with full scale data. Journal of Fluids Engineering, 107(1), 112-119.

Bark, G. (1986). Developmet of violent collapses in propeller cavitation. International Symposium on Cavitation and Multiphase Flow Noise, Proceedings of the ASME Symposium, Anaheim, CA, USA, 65-75.

Bark, G. (2000). Selected problems about scaling of cavitation noise at low and medium high frequencies. $34 t h$ WEGEMT School.

Bark, G., \& Berlekom, W. B. van. (1978). Experimental investigations of cavitation dynamics and cavitation noise. In Twelft Symposium on Naval Hydrodynamics (pp. 470-493). Washington, DC, USA.

Brooker, A., \& Humphrey, V. (2014). Measurement of Radiated Underwater Noise from a Small Research Vessel in Shallow Water. In A. Yücel Odabaşı Colloquium Series 1 st International Meeting - Propeller Noise \& Vibration 6th - 7th November 2014, Istanbul, Turkey.

Brown, N. A. (1976). Cavitation Noise Problems and Solutions. International Symposium on Shipboard Acoustics. Retrieved from http://trid.trb.org/view.aspx?id=70322

Fisher, F., \& Simmons, V. (1977). Sound absorption in sea water. The Journal of the Acoustical Society of America, 62, 
Hildebrand, J. A. (2005). Impacts of anthropogenic sound. In Marine mammal research: conservation beyond Crisis (pp. 101-124). JHU Press.

Hildebrand, J. A. (2009). Anthropogenic and natural sources of ambient noise in the ocean. Marine Ecology Progress Series, 395(5), 5-20. doi:10.3354/meps08353

IMO. (2011). Noise From Commerical Shipping and Its Adverse Impact on Marine Life- Development of an international standard for measurement of underwater noise radiated from merchant ships. MEPC 62nd session Agenda item 19. MEPC 62/19.

ITTC. (1987). Report of Cavitation Committee. Kobe, Japan, 18th International Towing Tank Conference, October.

ITTC. (2011). Cavitation Induced Pressure Fluctuations Model Scale Experiments. ITTC - Recommended Procedures and Guidelines. $7.5-0203$ - 03.3.

ITTC. (2014). Specialist Committee on Hydrodynamic Noise. In Final Report and Recommendations to the 27th ITTC (p. 45). Copenhagen, Sweden.

Korkut, E., \& Takinaci, A. C. (2013). 18M Research Vessel Wake Measurements. Istanbul Technical University Faculty Of Naval Architecture And Ocean Engineering February.

Latorre, R. (1982). TVC noise envelope-an approach to tip vortex cavitation noise scaling. Journal of Ship Research, 26(1), $65-75$.

McCormick, B. W. (1962). On Cavitation Produced by a Vortex Trailing from a Lifting Surface. Journal of Basic Engineering, 84, Transactions of ASME, 369-379.

McKenna, M. F., Ross, D., Wiggins, S., \& Hildebrand, J. A. (2012). Underwater radiated noise from modern commercial ships. Journal of Acoustical Society of America, 131(1), 92-103.

MCR. (2011). Cruise report for research projects conducted from R/V Song of the Whale in French and UK waters of the English Channel, 23, (September), 1-18.

MEPC. (2009). Noise From Commercial Shipping and Its Adverse Impacts on Marine Life. MEPC 60/18. 18 December 2009.

Okamura, N., \& Asano, T. (1988). Prediction of Propeller Cavitation Noise and Its Comparison with Full-Scale Measurements. Journal of Teh Society of Naval Architects of Japan, 164, 19-32.

Plesset, M. S. (1949). The Dynamics of Cavitation Bubbles. Journal of Applied Mechanics, 16(3), $277-282$.

Plesset, M. S., \& Prosperetti, A. (1977). Bubble dynamics and cavitation. Annual Review of Fluid Mechanics, 9(1), 145185 .

Raestad, A. (1996). Tip Vortex Index-an Engineering Approach to Propeller Noise Prediction. The Naval Architect, July/August, 11-16. Retrieved from http://trid.trb.org/view.aspx?id=480414

Renilson Marine Consulting Pty Ltd. (2009). Reducing underwater noise pollution from large commercial vessels, (March).

Ross, D. (1987). Mechanics of Underwater Noise. California, USA: Peninsula Publishing.

Shen, Y. T., \& Strasberg, M. (2003). The effect of scale on propeller tip-vortex cavitation noise. Hydromechanics Directorate Technical Report. Naval Surface Warfare Center Cerderock Division.

SONIC. (2012). Suppression Of underwater Noise Induced by Cavitation. FP7, Grant agreement no: 314394, Grant 
agreement no: 314394 .

2

Strasberg, M. (1977). Propeller cavitation noise after 35 years of study. ASME Symposium on Noise and Fluids Engineering. Retrieved from http://trid.trb.org/view.aspx?id=71740

Van der Graaf, A. J. ., Ainslie, M. A. ., André, M. ., Brensing, K. ., Dalen, J. ., Dekeling, R. P. A. ., ... Werner, S. . (2012). European Marine Strategy Framework Directive- Good Environmental Status (MSFD GES).

Wales, S. C., \& Heitmeyer, R. M. (2002). An ensemble source spectra model for merchant ship-radiated noise. The Journal of the Acoustical Society of America, 111(May 1999), 1211-1231. doi:10.1121/1.1427355

Wijngaarden, E. van. (2005). Recent developments in predicting propeller-induced hull pressure pulses. Proceedings of the 1st International Ship Noise and Vibration, 1-8.

\section{Appendix A. High speed cavitation observation videos}

The cavitation observation videos from both full-scale and cavitation tunnel tests are provided in this appendix. These videos for the corresponding conditions are vital as the main noise creating mechanism is the cavitation dynamics experienced. Hence, an enhanced insight can be achieved in terms of the differences between cavitation phenomenon present and their corresponding impact on the radiated noise level in comparative manner.

Click here to download Video: Condition 1 600RPM Full Scale High speed Video.avi

Video 1.: High speed cavitation observation video from tunnel tests for Condition 1

Click here to download Video: Condition 1 600RPM model Scale High speed Video.avi

\section{Video 2.: High speed cavitation observation video from full-scale trials for Condition 1}

Click here to download Video: Condition 2 900RPM Full Scale High speed Video.avi

\section{Video 3.: High speed cavitation observation video from tunnel tests for Condition 2}

Click here to download Video: Condition 2 900RPM Model Scale High speed Video.avi

Video 4.: High speed cavitation observation video from full-scale trials for Condition 2 
Click here to download Video: Condition 3 1200RPM Full Scale High speed Video.avi

Video 5.: High speed cavitation observation video from tunnel tests for Condition 3

Click here to download Video: Condition 3 1200RPM Model Scale High speed Video.avi

Video 6.: High speed cavitation observation video from full-scale trials for Condition 3

Click here to download Video: Condition 4 2000RPM Full Scale High speed Video.avi

Video 7.: High speed cavitation observation video from tunnel tests for Condition 4

Click here to download Video: Condition 4 2000RPM Model Scale High speed Video.avi

Video 8.: High speed cavitation observation video from full-scale trials for Condition 4 